\title{
Nonlinear elliptic boundary value problems versus their finite difference approximations: numerically irrelevant solutions*)
}

\author{
By H.O. Peitgen and D. Saupe at Bremen and K. Schmitt at Salt Lake City
}

\section{Introduction}

The aim of this paper is to continue and extend the discussion in [9] concerning the 'numerically irrelevant' solutions 'NIS' of finite difference approximations to certain nonlinear boundary value problems in the context of continuation methods or more generally and more appropriately, in the context of global topological perturbations of nonlinear eigenvalue problems. This latter technique as a numerical device for the global numerical study of nonlinear eigenvalue and bifurcation problems has been set up, discussed and applied to various nonlinear problems in [9] and [11]. Our discussion here will make an essential use of these techniques and ideas and moreover, provide a mathematical foundation for the numerical procedures suggested in [9] which were designed to avoid NIS. Our present attempt and approach has been motivated by a recent numerical study of Bohl [4] and a recent paper of Ambrosetti and Hess [3]. The former deals with the existence and characterization of NIS of finite difference approximations to a boundary value problem of the type

$$
u^{\prime \prime}+\mu \sin u=0, u(0)=0=u(\pi)
$$

whereas the latter is concerned with multiplicity results for positive solutions of asymptotically linear elliptic eigenvalue problems containing nonpositive nonlinearities.

The notion of NIS is by no means a precise one. It roughly expresses the fact that finite difference approximations to certain nonlinear eigenvalue problems may allow solutions which do not approximate solutions of the approximated differential equations, i.e., as the meshsize of the difference approximation is made smaller and smaller, these solutions do not approximate a solution of the problem at hand or they disappear. Envisaging the differential equation as a limit of these difference approximations it must be the case that the NIS must undergo a critical change.

*) This paper was written while the first author was a Visiting Professor at the University of Utah. 
More precisely, the first author, together with E. Allgower and K. Georg has developed the conceptual interpretation that NIS should be characterized in terms of singularities of certain embeddings of finite difference approximations with varying meshsize, where the meshsize is understood as a homotopy parameter.

Once one realizes that NIS may occur and one is engaged in a concrete algorithmic attempt to solve a given problem one must settle the important question whether a computed solution is "right" or "wrong" or one should design the algorithm in such a way to avoid the computation of NIS. This, of course, requires a good understanding of the occurrence of wrong solutions and their specific characterization.

An earlier paper of Allgower [1] has shown that the problem

$$
u^{\prime \prime}+\mu u^{k}=0, u(0)=0=u(\pi)
$$

may admit NIS for the finite difference approximation. Here the crucial point is that NIS do not have the symmetry properties which the solutions of (1.2) enjoy. Since the finite difference approximations also admit symmetric solutions those certainly are the candidates of NRS (numerically relevant solutions) and in fact this will be shown later. It is also shown in [1] that the existence of NIS is independent of the parameter $\mu$ and only depends upon the meshsize and, in fact, as the meshsize decreases, the NIS simply disappear. Bohl [4] recently observed the existence of a different type of NIS in his study of the pendulum equation (1.1). He found the existence of NIS which depend upon $\mu$ and the meshsize $h$ and which moreover, do have the symmetry properties of solutions of (1.1). Here the distinguishing feature between NIS and NRS is the uniqueness of positive solutions of (1.1). Thus it is immediate from the above discussion that in the presence of multiple solutions of a boundary value problem which do have the same structural type, the question of distinguishing between NIS and NRS becomes even more important the more involved the solution structure of a given problem.

One of the main themes of this paper is to present criteria which allow us to make such distinctions. We shall confine ourselves here to the study of problems of the type (1.1), (1.2) and further classes where multiplicity of positive solutions occur, a typical example of such a problem is

$$
u^{\prime \prime}+\mu f(u)=0, u(0)=0=u(\pi),
$$

where $f$ is an asymptotically linear or superlinear continuous function having several zeros.

This example has the property that for certain values of $\mu$, multiple positive solutions exist and that finite difference approximations indeed may possess NIS. In fact we show that for certain kinds of $f$, having $k$ positive zeros, at least $k^{n}$ ( $n=$ number of internal meshpoints) nontrivial solutions exist, almost all of which are NIS. In addition, we provide an understanding of these problems from an algorithmic point of view.

We interpret this phenomenon as follows. Let $A x=\lambda F(x)$ denote the finite difference approximation of a nonlinear boundary value problem where $A$ is the discretized differential operator. Then one may interpret $A$ as a coupling for the system of nonlinear equations determined by $F$. Since $\frac{1}{\lambda} A x=F(x)$, then for $\lambda$ large, the system 
would be practically uncoupled for bounded $x$, and hence would have as many solutions as there are zeros of $F$. This interpretation together with our results sheds new light onto coupled systems of differential equations which are parameter dependent.

The following sections of this paper are devoted to essentially three main topics.

(i) Existence of solutions of various boundary value problems and finite difference approximations thereof.

(ii) Existence of NIS and distinguishing between NIS and NRS.

(iii) Establishing numerical procedures which detect NRS and avoid NIS.

Since, again, our aim is to study phenomena, we have sacrificed generality (i.e., we do not discuss nonautonomous equations and restrict ourselves to ordinary rather than partial differential equations) and simply discuss some model equations where the arguments can be freed from much technical detail.

The organization of the paper is as follows: After some preliminary results and definitions, we provide some general criteria which may be used to detect NIS. We then study problems of the type (1.1) and (1.3) and their finite difference approximations and provide criteria for the calculation of solutions and at the same time obtain some simple criteria for bifurcation from $\infty$. We then present an analysis for the superlinear boundary value problem (1.2). Finally, we give numerical results.

All the numerical calculations were performed on the PDP 11-60 of the Mathematics Department of the University of Utah. The algorithms used are a package of interactive $P L$-algorithms which are designed for the global continuation of solutions of a $P L$ approximation of problems $H(\lambda, x)=0$, where $H: \mathbb{R} \times \mathbb{R}^{n} \rightarrow \mathbb{R}^{n}$ is continuous. The algorithms are implementations of ideas and procedures described in [9] and [11].

In what follows we often speak about generic properties of solutions of problems. This is to be understood in two different ways. (i) We consider solutions of $H(\lambda, x)=\bar{\varepsilon}$, where $H$ is of class $C^{\infty}$ and $\bar{\varepsilon}$ is a regular value and/or (ii) $\bar{\varepsilon}=\left(\varepsilon, \varepsilon^{2}, \ldots, \varepsilon^{n}\right)$ and $H_{T}(\lambda, x)=\bar{\varepsilon}$, where $H_{T}$ is a $P L$ approximation to $H$ subject to a given triangulation $T$ of $\mathbb{R}^{n+1}$ and $\varepsilon>0$ is small. The latter solutions are precisely those solutions exhibited in section 5 .

The phenomenon of NIS has been observed in other situations as well. We mention two further cases. Gaines [8] in his study of nonlinear boundary value problems by finite difference schemes found NIS which had the property that even though bounded, their first order divided differences became unbounded. $\mathrm{Li}$ and Yorke [10] observed that a difference approximation to the logistic equation $u^{\prime}=\alpha u(1-u)$ may be transformed into a first order difference equation of the form $x_{n+1}=\lambda x_{n}\left(1-x_{n}\right)$, an equation which for a certain parameter range will have 'chaotic' solutions, whereas the logistic equation is well behaved. ${ }^{1}$ ) As we shall see there exists a very similar phenomenon for boundary value problems as soon as nonlinearities have more than one zero. As already pointed out we have restricted ourselves to some simple examples which represent classes of problems which have received much attention in the literature, other types of nonlinearities will be considered at another time.

1) In this context H. Heatherly has pointed out to us the interesting paper by T. W. Chaundy and E. Phillips [The convergence of sequences defined by quadratic recurrence-formulae, Quart. J. of Math. (1936), 74-80]. 
Throughout we denote by $\mathbb{R}_{+}^{n}=\left\{x \in \mathbb{R}^{n}: x_{i} \geqq 0,1 \leqq i \leqq n\right\}$ and $\mathbb{R}_{+}^{n}=$ interior $\mathbb{R}_{+}^{n}$ and if $r>0$ then $B(r)=\left\{x \in \mathbb{R}^{n}:\|x\|<r\right.$, where $\left.\|x\|=\max _{1 \leqq i \leqq n}\left|x_{i}\right|\right\}$.

Acknowledgement. The first two authors thank the Department of Mathematics of the University of Utah for the very special hospitality during their visit and the generous availability of computer facilities. Thanks are also due to Frank Little for introducing and providing access to the computer aided geometric design package which was used in the graphics and authentic computer plots of our last section.

\section{Preliminary and auxiliary results}

In this section we consider the nonlinear system of equations

$$
A x=\lambda F(x),
$$

where $\lambda \in \mathbb{R}_{+}$and $F: \mathbb{R}^{n} \rightarrow \mathbb{R}^{n}$ is continuous and $A$ is an $n \times n$ matrix. The systems we have in mind arise as discretizations of nonlinear elliptic boundary value problems $L u=\lambda f(u, \nabla u)$ subject to linear homogeneous boundary conditions. For such systems it is often the case that a unique solution exists (examples will be seen later), whereas the discretization may have many solutions and the question arises which of these solutions ought to be taken as an approximation to the solution of the boundary value problem. The following lemma and its consequences show how such additional solutions arise.

Lemma 2. 1. Let $\mathcal{O}$ be an open bounded subset of $\mathbb{R}^{n}$ and let $F^{-1}(0) \cap \partial \mathcal{O}=\emptyset$. Assume that

$$
\operatorname{deg}(-F, \mathcal{O}, 0) \neq 0 \text {. }
$$

Then for all $\lambda>0$ sufficiently large (2. 1) has a solution $x_{\lambda} \in \mathcal{O}$.

Proof. Choose $\lambda_{*}$ so large that for $\lambda \geqq \lambda_{*}$

$$
\mu A x-F(x) \neq 0, x \in \partial \mathcal{O}, \mu=\frac{1}{\lambda} \in\left[0, \frac{1}{\lambda_{*}}\right] .
$$

Then

$$
\operatorname{deg}(\mu A-F, \mathcal{O}, 0)=\operatorname{deg}(-F, \mathcal{O}, 0)
$$

and thus

$$
\mu A x-F(x)=0
$$

has a solution in $\mathcal{O}$, proving the lemma.

Since (2. 2), of course, implies that $F$ has a zero in $\mathcal{O}$, we see that zeros of $F$ generate solutions of (2.1) for large $\lambda$.

Let us consider some special cases of Lemma 2.1.

Corollary 2. 2. Let $x_{0} \in \mathbb{R}^{n}$ be a zero of $F$ and let $F$ be of class $C^{1}$ in a neighborhood of $x_{0}$ and let $\operatorname{det} F^{\prime}\left(x_{0}\right) \neq 0$. Then for $\lambda$ sufficiently large (2.1) has a solution near $x_{0}$. 
Proof. Since $\operatorname{det} F^{\prime}\left(x_{0}\right) \neq 0$ it follows that $x_{0}$ is an isolated zero of $F$. Hence there exists a bounded open neighborhood $\mathcal{O}$ of $x_{0}$ such that $\overline{\mathcal{O}}$ contains no other zeros. But $\operatorname{deg}(-F, \mathcal{O}, 0)=(-1)^{n}$ sign det $F^{\prime}\left(x_{0}\right) \neq 0$. Hence, the result follows from Lemma 2.1.

Let $f: \mathbb{R} \rightarrow \mathbb{R}$ be a continuous function such that

$$
f^{-1}(0)=\left\{z_{1}, \ldots, z_{k}\right\}
$$

where $z_{1}<z_{2}<\cdots<z_{k}$, further assume that $f$ changes sign at these zeros, i.e. there exists $\varepsilon>0$ such that

$$
f(x) f(y)<0 \quad z_{i}-\varepsilon \leqq x<z_{i}<y \leqq z_{i}+\varepsilon, 1 \leqq i \leqq k .
$$

Let $F=\operatorname{col}\left(F_{1}, \ldots, F_{n}\right): \mathbb{R}^{n} \rightarrow \mathbb{R}^{n}$ be defined by

$$
F_{i}(x)=f\left(x_{i}\right), x=\operatorname{col}\left(x_{1}, \ldots, x_{n}\right)
$$

( $F$ will be called, as is customary, the Nemitskii operator associated with the scalar function $f$ ).

Corollary 2.3. Let $F$ be defined by (2.4). Then $F$ has $N=k^{n}$ zeros. And $F^{-1}(0)$ is given by

$$
F^{-1}(0)=\left\{w \in \mathbb{R}^{n}: w_{i} \in f^{-1}(0), 1 \leqq i \leqq n\right\} .
$$

Furthermore if $w=\left(w_{1}, \ldots, w_{n}\right)$ is such a zero and $\mathcal{O}$ is a bounded open neighborhood of $w$ with $(\overline{\mathcal{O}} \backslash\{w\}) \cap F^{-1}(0)=\emptyset$, then

$$
\operatorname{deg}(-F, \mathcal{O}, 0)= \pm 1
$$

Proof. $\operatorname{deg}(-F, \mathcal{O}, 0)$ is defined and by the Cartesian product formula and the excision property of degree

$$
\operatorname{deg}(-F, \mathcal{O}, 0)=\prod_{i=1}^{n} \operatorname{deg}\left(-f,\left(w_{i}-\varepsilon, w_{i}+\varepsilon\right), 0\right),
$$

and since $w_{i} \in f^{-1}(0)$ it follows from (2.3) that $\operatorname{deg}\left(-f,\left(w_{i}-\varepsilon, w_{i}+\varepsilon\right), 0\right)= \pm 1$, thus (2.6) follows. That there are $N=k^{n}$ such zeros follows by a simple counting argument.

Remark 2. 4. In the situation of Corollaries 2.2 and 2.3 the following observation is of importance. Let $w \in F^{-1}(0)$ and let $\mathcal{O}$ be a bounded open neighborhood of $w$ with $(\overline{\mathcal{O}} \backslash\{w\}) \cap F^{-1}(0)=\emptyset$. Let $\left\{x_{\lambda}\right\}$ be the collection of solutions of $(2.1)\left\{x_{\lambda}\right\} \subset \overline{\mathcal{O}}$. Then $x_{\lambda} \rightarrow w$ as $\lambda \rightarrow \infty$. This easily follows since

$$
\frac{1}{\lambda} A x_{\lambda}=F\left(x_{\lambda}\right)
$$

and thus since $\left\{A x_{\lambda}\right\}$ is bounded, and since every subset of $\left\{x_{\lambda}\right\}$ has a convergent subsequence converging to a zero of $F$ in $\overline{\mathcal{O}}$, i.e., $w$.

In addition to (2.3) let us now assume that for each $i, 1 \leqq i \leqq k$, there exist constants $m_{i}, q_{i}$ such that $m_{i} q_{i}>0$

(2. 8) $q_{i}(x-y) \leqq f(x)-f(y) \leqq m_{i}(x-y), \quad$ for $x, y \in\left[z_{i}-\varepsilon, z_{i}+\varepsilon\right], x \geqq y$.

We then obtain the following uniqueness result. 
Corollary 2. 5. Let $F$ be defined by (2.4) and let $f$ satisfy also (2.8). Then if $w \in F^{-1}(0)$ and $\lambda$ is sufficiently large there is a unique solution $x_{\lambda}$ of (2.1) such that $\left\|w-x_{\lambda}\right\|=\max _{i}\left|w_{i}-x_{\lambda i}\right|<\varepsilon$.

Proof. Existence of $x_{\lambda}$ has been verified, thus we only need to prove uniqueness. Let $w \in F^{-1}(0)$ and let $x, y$ be two solutions of (2.1) near $w$. Then

$$
\|F(x)-F(y)\|=\max _{i}\left|f\left(x_{i}\right)-f\left(y_{i}\right)\right| .
$$

But (2.8) implies that

$$
\left|f\left(x_{i}\right)-f\left(y_{i}\right)\right| \geqq \min \left\{\left|q_{i}\right|,\left|m_{i}\right|\right\}\left|x_{i}-y_{i}\right|
$$

i.e.

$$
\|F(x)-F(y)\| \geqq c\|x-y\|,
$$

where $c$ is some positive constant $\left(c\right.$ may be taken to equal $\left.\min _{i} \min \left(\left|q_{i}\right|,\left|m_{i}\right|\right)\right)$. On the other hand $\|F(x)-F(y)\| \leqq\left\|\frac{1}{\lambda} A(x-y)\right\| \leqq \frac{1}{\lambda}\|A\|\|x-y\|$, hence if $\lambda$ is so large that $\frac{1}{\lambda}\|A\|<c$, we conclude that $x=y$.

We shall now, for the remainder of the paper consider only nonlinear operators $F$ which are the Nemitskii operator of scalar functions $f$, as described above. In case one considers finite difference approximations to nonlinear boundary value problems where the nonlinearities are gradient dependent the equation $F(x)=0$, will no longer be uncoupled, however, Lemma 2.1 and Corollary 2. 2 still provide existence results for solutions of (2.1). Also if the gradient dependence is linear, these terms may be absorbed in $A$ and our results apply.

We now restrict attention to matrices $A$ which typically arise in the discretization of elliptic differential operators, namely $A=\left(a_{i j}\right)$ such that

(2.9) $\left\{\begin{array}{l}a_{i i}>0, a_{i j} \leqq 0, i \neq j, \\ a_{i i} \geqq \sum_{\substack{i=1 \\ j \neq i}}^{n}\left|a_{i j}\right|, \text { with strict inequality for at least one } i, 1 \leqq i, j \leqq n, \\ A \text { is irreducible. }\end{array}\right.$

The class of all such matrices shall be denoted by $\mathscr{M}$.

The following lemma gives some needed properties of matrices $A \in \mathscr{M}$ (see e.g. [14], [15]).

Lemma 2. 6. If $A \in \mathscr{M}$ then $A$ is nonsingular and

$$
A^{-1}\left(\mathbb{R}_{+}^{n} \backslash\{0\}\right) \subseteq \mathbb{R}_{+}^{n} .
$$

Furthermore $A$ has a unique positive eigenvalue $\lambda_{1}$ of multiplicity one and associated eigenvector $\bar{x} \in \mathbb{R}_{+}^{n}$. 
Lemma 2. 7. Let $A \in \mathscr{M}, f(0)=0$, and let there exist $\mu>0$ such that $f(x) \geqq-\mu x$, $x \in \mathbb{R}$. Then if $x$ is a solution of (2.1), it follows that $x \in \mathbb{R}_{+}^{n}$.

Proof. $A x=\lambda F(x) \Leftrightarrow(A+\lambda M) x=\lambda(F(x)+M x)$, where $M=\operatorname{diag}(\mu)$. But

$$
(A+\lambda M) \in \mathscr{M},
$$

hence

$$
x=\lambda(A+\lambda M)^{-1}(F(x)+M x) .
$$

But $(F+M)\left(\mathbb{R}^{n}\right) \cong \mathbb{R}_{+}^{n}$, hence by Lemma 2. 6, $x \in \mathbb{R}_{+}^{n}$.

Let us next define a class of asymptotically linear functions $\mathscr{F}$. We say $f \in \mathscr{F}$ if $f$ satisfies the following six conditions:

(i) $f$ is locally Lipschitz continuous.

(ii) There exists a $\mu>0$ such that $f(x) \geqq-\mu x$ for all $x<0$.

(iii) $f$ has zeros at $z_{0}=0<z_{1}<\cdots<z_{2 m}$

(iv)

(2.11) $\left\{\begin{array}{c}(-1)^{i} f(x)>0, x \in\left(z_{i}, z_{i+1}\right), i=0, \ldots, 2 m-1 \\ f(x)>0, x>z_{2 m}\end{array}\right.$

(v) There exist constants $q_{i}$ and $m_{i}, 1 \leqq i \leqq 2 m$ such that $(-1)^{i} q_{i},(-1)^{i} m_{i}>0$

(2. 12) $\quad q_{i}(x-y) \leqq f(x)-f(y) \leqq m_{i}(x-y), x \geqq y$, in a neighborhood of $z_{i}$.

(vi) There exist positive constants $m_{0}$ and $m_{\infty}$ such that

$$
\left\{\begin{array}{l}
f(x)=m_{0} x+o(x) \text { as } x \rightarrow 0+ \\
f(x)=m_{\infty} x+o(x) \text { as } x \rightarrow+\infty
\end{array}\right.
$$

We find it convenient to denote $f \in \mathscr{F}$ having $2 m+1$ zeros by $f_{m}$, i.e., $m$ denotes the number of positive (or negative) humps. A typical $f_{2}$ looks as follows:

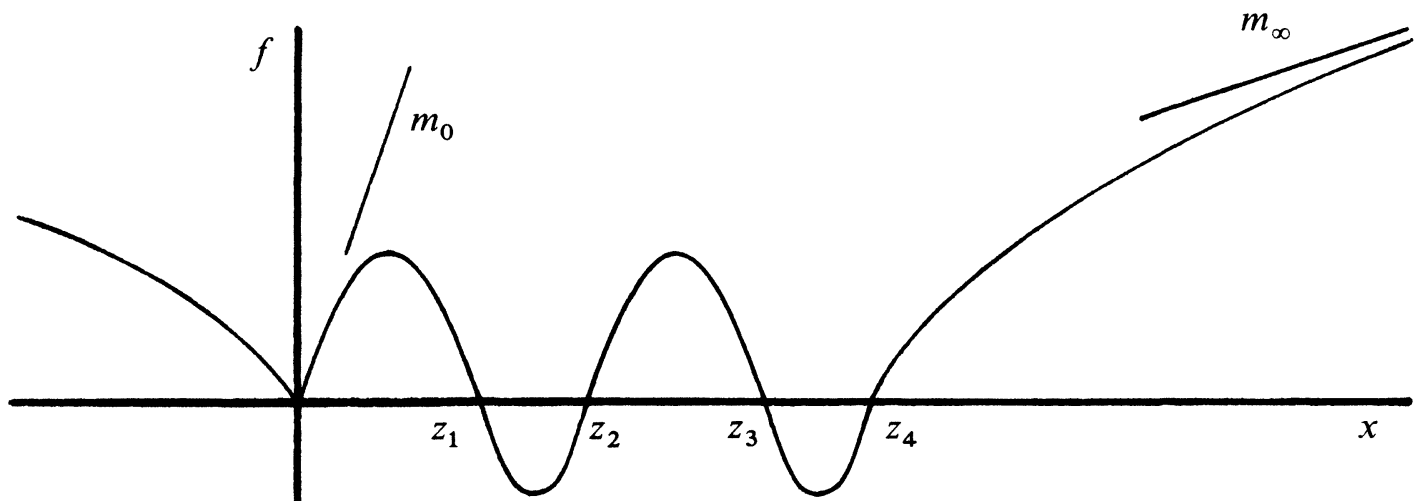


Lemma 2. 8. Let $A \in \mathscr{M}, f=f_{m} \in \mathscr{F}$. Let $x_{0}>0$ be such that $f\left(x_{0}\right)<0$. Then (2. 1) has no solution $x$ with $\|x\|=x_{0}$.

Proof. Let $x,\|x\|=x_{0}$ be a solution of (2. 1). It easily follows that Lemma 2.7 may be applied to conclude that $x \in \mathbb{R}_{+}^{n}$. Hence since $\|\cdot\|$ is monotone with respect to the partial ordering induced by $\mathbb{R}_{+}^{n}$, it follows that $x_{i} \leqq x_{0}, 1 \leqq i \leqq n$. Choose $i$ such that $x_{i}=x_{0}$. Then

$$
a_{i i} x_{i}+\sum_{\substack{i=1 \\ j \neq i}}^{n} a_{i j} x_{j}=\lambda f\left(x_{0}\right)<0
$$

since $x_{j} \leqq x_{i}$ and $A \in \mathscr{M}$ we get

$$
x_{i} \sum_{j=1}^{n} a_{i j} \leqq \lambda f\left(x_{0}\right)<0
$$

contradicting $x_{i} \geqq 0$ and $\sum_{j=1}^{n} a_{i j} \geqq 0$.

Remark 2. 9. The conclusion of the lemma remains valid in case $f\left(x_{0}\right)=0$. The proof is not significantly different in this case.

Remark 2. 10. The above sequence of results implies that all nontrivial solutions $x$ of (2.1) must lie in $\mathbb{R}_{+}^{n}$ and are such that $\|x\| \in\left(z_{i}, z_{i+1}\right)$, i even or $\|x\|>z_{2 m}$, for each $f_{m} \in \mathscr{F}$.

The next couple of lemmas and remarks will be of aid in establishing the general bifurcation results of the next section and further will permit necessary estimates for the global perturbation and continuation of solutions. We here follow to some extent the ideas of [3].

Lemma 2. 11. Let $A \in \mathscr{M}, f \in \mathscr{F}$ and let $\Lambda$ be a compact real interval with $\lambda_{\infty}=\frac{\lambda_{1}}{m_{\infty}} \notin \Lambda$. Then there exists $R>0$ such that $\|x\| \geqq R$ implies $A x \neq \lambda F(x)$, for $\lambda \in \Lambda$.

Proof. Assume there are sequences $\left\{x_{m}\right\},\left\|x_{m}\right\| \rightarrow \infty,\left\{\lambda_{m}\right\} \cong \Lambda$ such that

$$
A x_{m}=\lambda_{m} F\left(x_{m}\right)
$$

Hence $x_{m} \in \mathbb{R}_{+}^{n}$. We write $F(x)=m_{\infty} x+\phi(x)$, where $\phi(x)=o(\|x\|)$ as $\|x\| \rightarrow \infty$. Then letting $y_{m}=\frac{x_{m}}{\left\|x_{m}\right\|}$ we may assume that $y_{m} \rightarrow y \in \mathbb{R}_{+}^{n} \backslash\{0\}$, and $\lambda_{m} \rightarrow \lambda \in \Lambda$. Thus, since

$$
A y_{m}=\lambda_{m} m_{\infty} y_{m}+\lambda_{m} \frac{\phi\left(x_{m}\right)}{\left\|x_{m}\right\|}
$$

one gets

$$
A y=\lambda m_{\infty} y .
$$

Therefore, by Lemma $2.6, y \in \stackrel{\circ}{R}_{+}^{n}$ and $\lambda m_{\infty}=\lambda_{1}$, i.e., $\frac{\lambda_{1}}{m_{\infty}} \in \Lambda$, a contradiction. 
Remark 2. 12. Lemma 2.11 implies that $\lambda_{\infty}=\frac{\lambda_{1}}{m_{\infty}}$ is the only possible point of bifurcation from infinity.

Using arguments similar to the ones above we obtain the following resuit.

Lemma 2. 13. Let $A \in \mathscr{M}, f \in \mathscr{F}$ and let $\Lambda$ be a compact real interval with $\lambda_{0}=\frac{\lambda_{1}}{m_{0}} \notin \Lambda$. Then there exists $r>0$ such that $0<\|x\| \leqq r$ implies $A x \neq \lambda F(x)$ for $\lambda \in \Lambda$.

Remark 2. 14. It follows from Lemma 2.13 that $\lambda_{0}$ is the only possible point of bifurcation from 0 .

Remark 2. 15. (i) In case $f$ is superlinear at $\infty$, i.e., $\lim _{x \rightarrow \infty} \frac{f(x)}{x}=\infty$, the previous set of lemmas hold when $\lambda_{\infty}$ is replaced by $\lambda_{\infty}=0$. (ii) Often it is desirable to weaken the differentiability assumptions (2.13) to the following type of Lipschitz conditions: There exist positive constants $m_{01}, m_{02}, m_{\infty 1}, m_{\infty 2}$ such that

(2. 14) $\begin{cases}m_{01} x+o(x) \leqq f(x) \leqq m_{02} x+o(x) & \text { as } x \rightarrow 0+, \\ m_{\infty 1} x+o(x) \leqq f(x) \leqq m_{\infty 2} x+o(x) & \text { as } x \rightarrow+\infty .\end{cases}$

For such nonlinearities bifurcation need not take place from isolated points but rather will take place from intervals, see e.g., [13]. In fact using the ideas of [13] one may establish the following facts for functions $f \in \mathscr{F}$ which satisfy (2.14) rather than (2.13):

Let $\Lambda$ be a compact interval such that $\left[\frac{\lambda_{1}}{m_{\infty 2}}, \frac{\lambda_{1}}{m_{\infty 1}}\right] \cap \Lambda=\emptyset$, then there exists a constant $R$ such that $\|x\| \geqq R$ implies $A x \neq \lambda F(x), \lambda \in \Lambda$, and in fact the bifurcation points from infinity must be contained in $\left[\frac{\lambda_{1}}{m_{\infty 2}}, \frac{\lambda_{1}}{m_{\infty 1}}\right]$. And dually if $\Lambda \cap\left[\frac{\lambda_{1}}{m_{02}}, \frac{\lambda_{1}}{m_{01}}\right]=\emptyset$ one can find a constant $r>0$ such that for $\lambda \in \Lambda$, and $0<\|x\| \leqq r$ implies $A x \neq \lambda F(x)$ and one can assert that bifurcation from zero can only take place in

$$
\left[\frac{\lambda_{1}}{m_{02}}, \frac{\lambda_{1}}{m_{01}}\right] \text {. }
$$

Lemma 2. 16. Let $A \in \mathscr{M}$ and $f \in \mathscr{F}$ be such that $f(x) \geqq \alpha x, \alpha>0, x>0$. Then there exists $\lambda_{*}$ such that if $\lambda>\lambda_{*}$, (2.1) cannot have any solutions $x \in \mathbb{R}_{+}^{n} \backslash\{0\}$.

Proof. Assume the contrary, then we obtain a sequence $\left\{\lambda_{m}\right\}, \lambda_{m} \rightarrow \infty$ and $\left\{x_{m}\right\} \subseteq \mathbb{R}_{+}^{n} \backslash\{0\}$ such that

$$
A x_{m}=\lambda_{m} F\left(x_{m}\right) \geqq \lambda_{m} \alpha x_{m},
$$

and since $A^{-1}$ is a positive operator (Lemma 2.6)

$$
y_{m}=\frac{x_{m}}{\left\|x_{m}\right\|} \geqq \lambda_{m} \alpha A^{-1} y_{m} .
$$

Again, we may assume that $y_{m} \rightarrow y \in \mathbb{R}_{+}^{n} \backslash\{0\}$, and thus, since $\frac{1}{\lambda_{m}} y_{m} \rightarrow 0$, we obtain $A^{-1} y=0$, a contradiction to $A^{-1}$ being nonsingular. 
Remark 2. 17. In case $A$ is symmetric we may use Lemma 2.6 to estimate $\lambda_{*}$. In fact for $x \in \mathbb{R}_{+}^{n}$

Thus since

$$
A x=\lambda F(x) \geqq \lambda \alpha x .
$$

we get

$$
A \bar{x}=\lambda_{1} \bar{x},
$$

$$
(A x, \bar{x})=(x, A \bar{x})=\left(x, \lambda_{1} \bar{x}\right)=\lambda_{1}(x, \bar{x})
$$

$\left((\right.$,$\left.) is the scalar product of \mathbb{R}^{n}\right)$, but

$$
(A x, \bar{x}) \geqq(\lambda \alpha x, \bar{x})=\lambda \alpha(x, \bar{x}),
$$

hence since $\bar{x}, x \in \mathbb{R}_{+}^{n} \backslash\{0\}$, it follows that

$$
\lambda_{1} \geqq \lambda \alpha \text {, i.e., } \lambda \leqq \frac{\lambda_{1}}{\alpha}=\lambda_{*} .
$$

\section{Existenice of NIS and NRS - continuation results}

In this section we consider finite difference approximations to nonlinear elliptic equations $L u+\mu f(u)=0$ subject to homogeneous linear boundary constraints.

For example, in the case of problem (1.3) one divides $[0, \pi]$ into $n+1$ equal parts of meshsize $\frac{\pi}{n+1}=h$ and one replaces $u^{\prime \prime}$ by $\frac{u((i+1) h)-2 u(i h)+u((i-1) h)}{h^{2}}$. Letting $u(i h)=x_{i}$, then since $x_{0}=0=x_{n+1}$, we obtain the difference scheme

$$
\left\{\begin{aligned}
2 x_{1}-x_{2} & =h^{2} \mu f\left(x_{1}\right), \\
-x_{1}+2 x_{2}-x_{3} & =h^{2} \mu f\left(x_{2}\right), \\
\vdots & \vdots \\
-x_{n-1}+2 x_{n} & h^{2} \mu f\left(x_{n}\right),
\end{aligned}\right.
$$

which we write as

$$
A x=\lambda F(x), \lambda=h^{2} \mu,
$$

where $A$ is the symmetric $n \times n$ matrix

$$
A=\left\{\begin{array}{rrrrr}
2 & -1 & & & \\
-1 & 2 & -1 & & 0 \\
0 & \cdot & \cdot & \cdot & \cdot \\
0 & & -1 & 2 & -1 \\
& & & -1 & 2
\end{array}\right\}
$$

and

$$
F(x)=\operatorname{col}\left(f\left(x_{i}\right)\right), x=\left(x_{1}, \ldots, x_{n}\right) .
$$

It is well known that $A \in \mathscr{M}$. Again we emphasize that more general $A$ are permissible in what follows (i.e., the requirement that $A \in \mathscr{M}$ suffices). Let $\lambda_{1}$ be the unique positive eigenvalue whose existence follows from Lemma 2. 6. 
Much of the discussion to follow is equally valid for different types of homogeneous boundary conditions, e.g., if instead of (1.3) we consider the problem

$$
u^{\prime \prime}+\mu f(u)=0, u(0)=0=u^{\prime}\left(\frac{\pi}{2}\right)
$$

then we obtain as a finite difference approximation a problem of type (3.2) with $A$ given by

$$
A=\left(\begin{array}{rrrrrr}
2 & -1 & 0 & \ldots & \ldots & 0 \\
-1 & 2 & -1 & & & \vdots \\
\vdots & & & & 0 \\
0 & \ldots & \ldots & -1 & 2 & -1 \\
0 & \ldots & \ldots & \ldots & -2 & 2
\end{array}\right)
$$

where again $A \in \mathscr{M}$. Since solutions of (1.3) will be symmetric it is natural to consider such boundary value problems and use the symmetric extension (about $\pi / 2$ ) as a solution of (1. 3).

Concerning (3. 2) we establish the following theorem.

Theorem 3. 1. Let $f=f_{m} \in \mathscr{F}$. Then the following are true:

(a) For all $m \geqq 0, \lambda_{0}=\frac{\lambda_{1}}{m_{0}}$ is a bifurcation point from zero for (3.2) from which an unbounded continuum $\Sigma_{0}$ of nontrivial solutions of (3.2) emanates, and $\lambda_{\infty}=\frac{\lambda_{1}}{m_{\infty}}$ is $a$ bifurcation point from infinity with a corresponding branch $\Sigma_{\infty}$ of nontrivial solutions.

(b) If $m=0$, then $\Sigma_{0}=\Sigma_{\infty}$ and $(\lambda, x) \in \Sigma_{0}$ implies $\lambda \leqq \lambda_{*}$, where $\lambda_{*}$ is given by Remark 2. 17.

(c) If $m \geqq 1$, then $\Sigma_{0} \cap \Sigma_{\infty}=\emptyset$, more precisely

$$
\begin{cases}(\lambda, x) \in \Sigma_{0} \Rightarrow\|x\|<z_{1} & \text { and } \lambda \rightarrow \infty \\ (\lambda, x) \in \Sigma_{\infty} \Rightarrow\|x\|>z_{2 m} & \text { and } \lambda \rightarrow \infty,\end{cases}
$$

in either case there exists $\varepsilon>0$ such that $\lambda \geqq \varepsilon$. There are no solutions $x$ of (3.2) with $z_{2 i-1} \leqq\|x\| \leqq z_{2 i}$.

(d) $\Sigma_{0}, \Sigma_{\infty} \subseteq \mathbb{R}_{+}^{n}$.

Using Corollary 2. 3 and Corollary 2.5 we obtain a unique solution of (3.2) in the neighborhood of every zero $w$ of $F$ with $w \in \mathbb{R}_{+}^{n}$ for all large $\lambda$ and thus many other solutions of (3.2) must be expected. We describe this in the following theorem.

Theorem 3. 2. Let $f=f_{m} \in \mathscr{F}$, then for all large $\lambda$, (3.2) has at least $N=(2 m)^{n}$ solutions in $\stackrel{\circ}{R}_{+}^{n}$; these solutions may be found close to the zeros of $F$ which lie in $\stackrel{\circ}{R}_{+}^{n}$. Furthermore, the solutions of (3.2) have the following properties: 
(i) For each $k=1, \ldots, 2 m$ there are $k^{n}-(k-1)^{n}$ solutions (whose local degree is odd) with norm close to $z_{k}$ (for large $\lambda$ ).

(ii) If $v\left(z_{i}, z_{i+1}\right)$ denotes the number of $\mathbb{R}_{+}^{n}$ solutions in $\overline{B\left(z_{i+1}\right)} \backslash B\left(z_{i}\right), i=1, \ldots, 2 m$ (where $z_{2 m+1}=\infty$ ) (whose local degree is odd), then (for large $\lambda$ )

$$
\begin{aligned}
& v\left(z_{i}, z_{i+1}\right)=\left\{\begin{array}{cc}
(i+1)^{n}-(i-1)^{n}, & i \geqq 2, \text { i even, } \\
1 & i=0, \\
0 & , i \text { odd, }
\end{array}\right. \\
& v\left(z_{2 m}, \infty\right)=(2 m)^{n}-(2 m-1)^{n} .
\end{aligned}
$$

(iii) Generically there exist at least $\frac{1}{2}(N-2)$ nontrivial continua $\Sigma_{i}$, which are unbounded in the $\lambda$ direction, and there exists $\bar{\lambda}>0$ such that:

at least $\frac{v\left(z_{k}, z_{k+1}\right)}{2}$ such are contained in $[\bar{\lambda}, \infty) \times B\left(z_{k+1}\right) \backslash \bar{B}\left(z_{k}\right), k$ even, and there are at least $\frac{1}{2} v\left(z_{2 m}, \infty\right)-1$ such in $[\bar{\lambda}, \infty) \times \mathbb{R}^{n} \backslash \bar{B}\left(z_{2 m}\right)$. Each of these $\Sigma_{i}$, $1 \leqq i \leqq \frac{1}{2}(N-2)$ are generically homeomorphic to a folded real line.

Proof. Using Corollary 2. 3 and Corollary 2.5 we deduce the existence of a solution of (3.2) in small neighborhoods of zeros $w \in \mathbb{R}_{+}^{n}$ of $F$ for all $\lambda$ sufficiently large. There are $(2 m)^{n}=N$ such zeros. That 0 is an isolated solution follows since large $\lambda$ are not eigenvalues of $A$. We may identify each $\mathbb{R}_{+}^{n}$ zero of $F$ with a lattice point in $\mathbb{Z}_{+}^{n}$, (i) and (ii) therefore follow by counting such lattice points and using Lemma 2. 8. Assertion (iii) follows from the above arguments, the fact that for $\lambda<\varepsilon$ no positive solutions exist, the Leray-Schauder continuation method and Theorem 3. 3 to follow.

We remark at this point that there may be other solutions $x_{\lambda}$ of (3.2), for $\lambda$ large, namely solutions close to zeros $w$ of $F, w \in \partial \mathbb{R}_{+}^{n}$, i.e., those zeros of $F$ having at least one of their components zero. ${ }^{2}$ ) Since other solutions will be seperated from such and since each such zero $w$ is isolated we may compute $\operatorname{deg}(A-\lambda F, \mathcal{O}, 0)$, where $\mathcal{O}$ is a small neighborhood of $w$. We claim that $\operatorname{deg}(A-\lambda F, \mathcal{O}, 0)=0$. It follows from our consideration that

$$
d=\operatorname{deg}(A-\lambda F, \mathcal{O}, 0)=\operatorname{deg}(\mu A-F, \mathcal{O}, 0), \quad 0 \leqq \mu \leqq \frac{1}{\lambda_{*}},
$$

$\lambda_{*}$ large, and thus by homotopy invariance $d=\operatorname{deg}(-F, \mathcal{O}, 0)$. Let $w=\left(w_{1}, \ldots, w_{n}\right)$, then for each $j, 1 \leqq j \leqq n, w_{j}=z_{i}$ for some $i, 0 \leqq i \leqq 2 m$, with at least one $w_{j}=0$. It follows from the Cartesian product formula of Brouwer degree that

$$
\operatorname{deg}(-F, \mathcal{O}, 0)=\prod_{j=1}^{n} \operatorname{deg}\left(-f, \mathcal{O}_{j}, 0\right)
$$

$\left.{ }^{2}\right)$ If $A$ is the discretization corresponding to (1.3) then an easy argument shows that $A x-\lambda F(x)=0$ and $x \in \partial R_{+}^{n}$ implies that $x \equiv 0$. 
where $\mathcal{O}_{j}$ is a small neighborhood of $w_{j} \in \mathbb{R}$, however, for those $j$ where $w_{j}=0$, $\operatorname{deg}\left(-f, \mathcal{O}_{j}, 0\right)=0$ (recall that $f(x)>0, x \neq 0$ in small neighborhoods of 0 ), hence $d=0$. As a special case we have, of course also shown that

$$
\operatorname{deg}(A-\lambda F, B(\varepsilon), 0)=0
$$

for all $\lambda$ sufficiently large and $\varepsilon>0$ sufficiently small.

The differentiability assumptions at 0 and $\infty$ may be relaxed to conditions of the type (2.14) in which case we obtain a result similar to Theorem 3.1 except that in part (a) the bifurcation points $\lambda_{0}$ and $\lambda_{\infty}$ will be replaced by bifurcation intervals $\left[\frac{\lambda_{1}}{m_{02}}, \frac{\lambda_{1}}{m_{01}}\right]$ and $\left[\frac{\lambda_{1}}{m_{\infty 2}}, \frac{\lambda_{1}}{m_{\infty 1}}\right]$. Arguments like the ones used here combined with ideas from [13] would establish these claims.

We now define a global perturbation $\Phi: \mathbb{R} \times \mathbb{R}^{n} \rightarrow \mathbb{R}^{n}$ of (3.2) in order to provide (i) a numerical access to $\Sigma_{\infty}$ via $\Sigma_{0}$ in case $m \geqq 1$ and (ii) to prove (a) of Theorem 3. 1 .

The idea is to perturb (3.2) in such a way that the disjoint continua $\Sigma_{0}$ and $\Sigma_{\infty}$ become connected, while the continua $\Sigma_{i}$ either disappear or close up to homeomorphs of $S^{1}$, i.e., no longer interfere with $\Sigma_{0}$ and $\Sigma_{\infty}$. More precisely $\Phi$ is chosen such that $\Phi^{-1}(0)$ is generically a union of a real line (representing $\tilde{\Sigma}_{0}=\tilde{\Sigma}_{\infty}$ ) and finitely many $S^{1}$ (representing the $\tilde{\Sigma}_{i}$ ) (see figure 3 ).

To achieve that goal we define for $f_{m} \in \mathscr{F}, m>1$,

$$
f_{1}(x)=\left\{\begin{array}{l}
f_{m}(x), x \leqq z_{1}, z_{2 m} \leqq x \\
g(x), z_{1} \leqq x \leqq z_{2 m}
\end{array}\right.
$$

where $g\left(z_{1}\right)=g\left(z_{2 m}\right)=0$ and $g$ is so chosen that $f_{1} \in \mathscr{F}$ (i.e., $f_{1}$ satisfies the requirements (2.11)-(2.13) for $m=1)$.
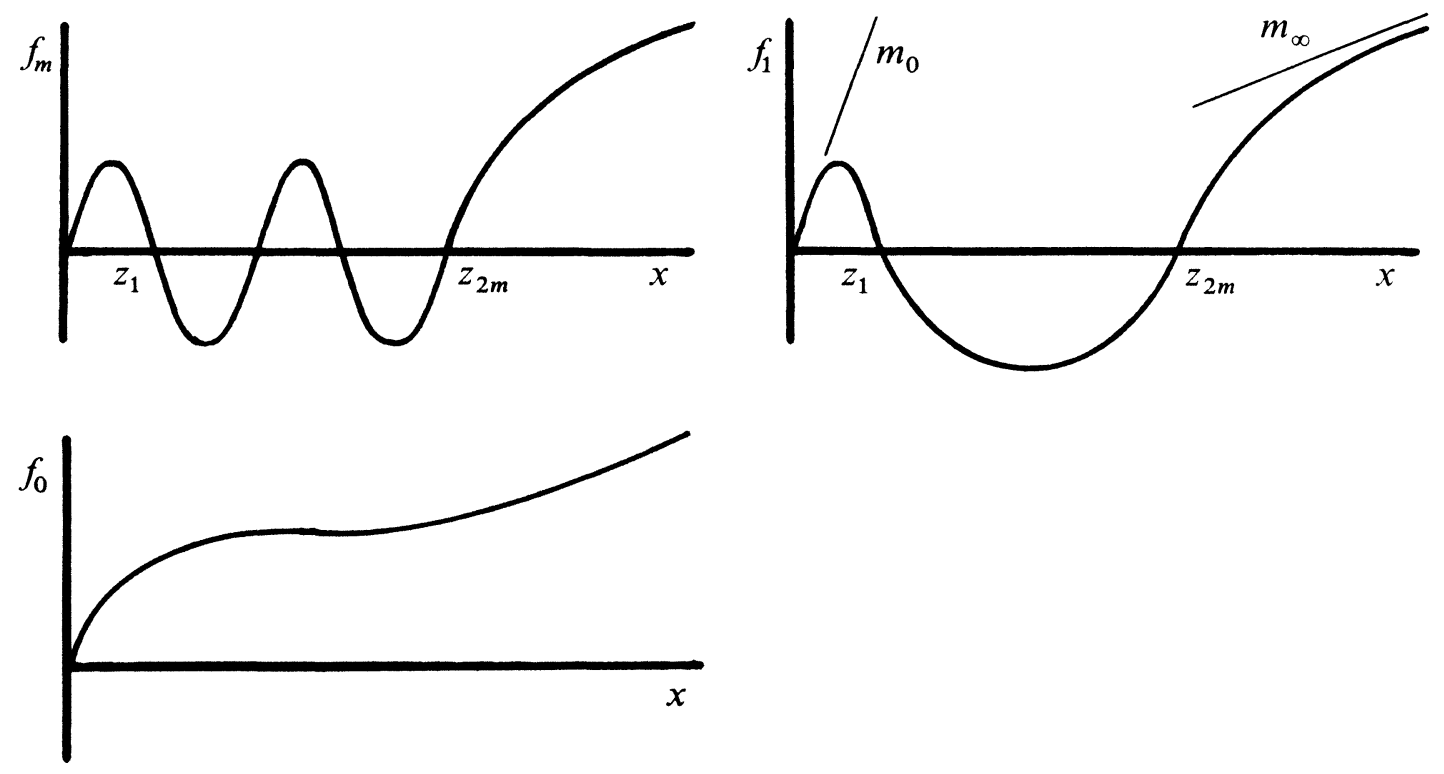

Figure 2 
Let $f_{0} \in \mathscr{F}$ (i.e., $f_{0}$ satisfies the requirements (2.11)-(2.13) $m=0$ ) be such that $f_{0}=f_{m}$ in a neighborhood of 0 and of $\infty$.

As perturbation parameters we choose constants $\mu_{1}, \ldots, \mu_{4}$ such that

$$
\max \left\{\lambda_{0}, \lambda_{\infty}\right\}<\mu_{1}<\mu_{2}<\mu_{3}<\mu_{4}<+\infty,
$$

where $\lambda_{*}<\mu_{4}$, and where $\lambda_{*}$ is given by Remark 2.17 for $f_{0}$. We define

$$
\left\{\begin{array}{l}
h_{1}(\lambda, x)=\frac{\lambda-\mu_{2}}{\mu_{1}-\mu_{2}} f_{m}(x)+\frac{\lambda-\mu_{1}}{\mu_{2}-\mu_{1}} f_{1}(x), \mu_{1} \leqq \lambda \leqq \mu_{2}, \\
h_{2}(\lambda, x)=\frac{\lambda-\mu_{4}}{\mu_{3}-\mu_{4}} f_{1}(x)+\frac{\lambda-\mu_{3}}{\mu_{4}-\mu_{3}} f_{0}(x), \mu_{3} \leqq \lambda \leqq \mu_{4} .
\end{array}\right.
$$

Observe that

$$
\begin{aligned}
h_{i}(\lambda, x) & =m_{0} x+o(x) \quad \text { as } x \rightarrow 0+, \\
& =m_{\infty} x+o(x) \quad \text { as } x \rightarrow+\infty, i=1,2 .
\end{aligned}
$$

Denote by $F_{m}, F_{1}, F_{0}, H_{1}, H_{2}$ the associated nonlinear Nemitskii operators. Put

$$
G(\lambda, x)=\left\{\begin{array}{lr}
\lambda F_{m}(x), & \lambda \leqq \mu_{1}, \\
H_{1}(\lambda, x), & \mu_{1} \leqq \lambda \leqq \mu_{2}, \\
\lambda F_{1}(x), & \mu_{2} \leqq \lambda \leqq \mu_{3}, \\
H_{2}(\lambda, x), & \mu_{3} \leqq \lambda \leqq \mu_{4}, \\
\lambda F_{0}(x), & \mu_{4} \leqq \lambda,
\end{array}\right.
$$

and set

$$
\Phi(\lambda, x)=x-A^{-1} G(\lambda, x) .
$$

The following result will describe the solution set of the perturbed problem

$$
\Phi(\lambda, x)=0 .
$$

Theorem 3. 3. $\Phi^{-1}(0)$ contains continua $\tilde{\Sigma}_{0}=\tilde{\Sigma}_{\infty}$ and $\tilde{\Sigma}_{i}, 1 \leqq i \leqq k, k \leqq \frac{1}{2}(N-2)$ where $\tilde{\Sigma}_{0}$ bifurcates from 0 at $\lambda_{0}$ and from infinity at $\lambda_{\infty}$, and the $\tilde{\Sigma}_{i}$ satisfy the conditions of the $\Sigma_{i}$ of Theorem 3.2, ((i), (ii)). Those continua $\tilde{\Sigma}_{i}$ which lie in $B\left(z_{2 i+1}\right) \backslash \overline{B\left(z_{2 i}\right)}$ are generically homeomorphic to a 1-sphere and $\tilde{\Sigma}_{0}=\tilde{\Sigma}_{\infty}$ is generically homeomorphic to a real line.

Remark 3.4 (Numerical Interpretation). (i) Under appropriate regularity assumptions (e.g. $f_{m}(x)=m_{0} x+\phi_{0}(x)$ near $0, \phi_{0}$ is smooth, $\phi_{0}(x)=o(x), f_{m}(x)=m_{\infty} x+\phi_{\infty}(x)$ near $+\infty, \phi_{\infty}$ is smooth and $\phi_{\infty}(x)=o(x)$ as $x \rightarrow \infty$ ) the simplicity of $\lambda_{0}$ (respectively $\lambda_{\infty}$ ) will imply that $\tilde{\Sigma}_{0}$ (respectively $\tilde{\Sigma}_{\infty}$ ) are locally unique. More precisely the theory of Crandall and Rabinowitz [12] will imply that in a small neighborhood of $\left(\lambda_{0}, 0\right), \tilde{\Sigma}_{0}$ is a parametrizable curve containing all small nontrivial solutions. The appropriate description of $\tilde{\Sigma}_{\infty}$ near infinity is obtained from the classical transformations

$$
x \rightarrow z:=\|x\|^{-2} x, A x=\lambda F_{m}(x) \rightarrow A z=\lambda\|z\|^{2} F_{m}\left(\frac{z}{\|z\|^{2}}\right) .
$$


(ii) As demonstrated in the discussion of Figures 25 and 26 in section 5 it may happen that the perturbation $\Phi(\lambda, x)=0$ creates additional singularities: There it is shown that the $\tilde{\Sigma}_{0}=\tilde{\Sigma}_{\infty}$ component in $\Phi^{-1}(0)$ may undergo a secondary bifurcation to NIS components $\Sigma_{i}$ of problem (3. 2). In view of this it is important to note that (cf. [11], Theorem 9.1.3) in our situation also generically global bifurcation occurs, i.e. $\tilde{\Sigma}_{0}=\tilde{\Sigma}_{\infty}$ is generically homeomorphic to a real line. More precisely, it may happen that in the course of the perturbation $\tilde{\Sigma}_{0}=\tilde{\Sigma}_{\infty}$ picks up some $\Sigma_{i}$ components of problem (3.2) (which may be NIS to problem (1.3)).

(iii) Local uniqueness (cf. (i)) of $\Sigma_{0}$ and $\Sigma_{\infty}$ and remark (ii) may be interpreted by concluding that $\Sigma_{0}$ and $\tilde{\Sigma}_{0}$ (resp. $\Sigma_{\infty}$ and $\tilde{\Sigma}_{\infty}$ ) consist of NRS to problem (1.3) near the bifurcation point from zero (resp. infinity). Thus, any algorithm which is designed to globally solve the generic problem (e.g. a $P L$ or smooth continuation algorithm) associated with $\Phi(\lambda, x)=0$, will provide an access to the NRS component via $\tilde{\Sigma}_{0}=\tilde{\Sigma}_{\infty}$ from the trivial solutions.

(iv) As the proof of Theorem 3. 3 will show, we have a way of detecting bifurcation from $\infty$ simply through bifurcation from $0\left(\tilde{\Sigma}_{0}=\tilde{\Sigma}_{\infty}\right)$, thus avoiding elaborate degree or index calculations in a neighborhood of $\infty$, a method of proof very much different from that used in [3].

Proof of Theorem 3. 3. Using the results of section 2 and the special construction of $G(\lambda, x)$ we may construct regions $X^{i}, Y^{i}$ as follows:

For each $\delta_{0}^{+}>0$, there exists $r_{\delta_{0}}^{+}>0$ such that

$$
\Phi(\lambda, x) \neq 0, \text { for }(\lambda, x) \in\left[\lambda_{0}+\delta_{0}^{+}, \infty\right) \times \overline{B\left(r_{\delta_{0}}^{+}\right)}=X^{1},
$$

(see Lemma 2.13 and Lemma 2.16).

For each $\delta_{\infty}^{+}>0$, there exists $R_{\delta_{\infty}^{+}}$such that

(3. 10) $\Phi(\lambda, x) \neq 0$, for $(\lambda, x) \in\left[\lambda_{\infty}+\delta_{\infty}^{+}, \infty\right) \times \mathbb{R}^{n} \backslash B\left(R_{\delta_{\infty}^{+}}\right)=X^{2}$,

(see Lemma 2.11 and Lemma 2.16).

$$
\Phi(\lambda, x) \neq 0, \text { for }(\lambda, x) \in\left[\mu_{4}, \infty\right) \times \mathbb{R}^{n}=X^{3},
$$

(see Remark 2.17 and definition of $\Phi$ ).

$$
\Phi(\lambda, x) \neq 0 \text {, for }(\lambda, x) \in\left[0, \mu_{2}\right] \times \overline{B\left(z_{2 i}\right)} \backslash B\left(z_{2 i-1}\right)=Y^{i}, 1 \leqq i \leqq m,
$$

(see Lemma 2. 8 and Remark 2.9).

$$
\Phi(\lambda, x) \neq 0 \text {, for }(\lambda, x) \in\left[\mu_{2}, \mu_{3}\right] \times \overline{B\left(z_{2 m}\right)} \backslash B\left(z_{1}\right)=X^{4},
$$

(see Lemma 2. 8 and Remark 2.9).

$$
\Phi(\lambda, x) \neq 0, \text { for }(\lambda, x) \in[0, \varepsilon] \times\left(\mathbb{R}^{n} \backslash\{0\}\right)=X^{5} .
$$

For each $\delta_{\infty}^{-}>0$, there exists $R_{\delta_{\infty}^{-}}>0$ such that

$$
\Phi(\lambda, x) \neq 0 \text {, in }\left[0, \lambda_{\infty}-\delta_{\infty}^{-}\right] \times \mathbb{R}^{n} \backslash B\left(R_{\delta_{\infty}}\right)=X^{6}
$$

(see Lemma 2. 11).

Since $A$ is nonsingular it follows that for $\lambda>0, \varepsilon>0$ small enough

$$
\operatorname{deg}\left(A-\lambda F_{m}, B(\varepsilon), 0\right)=\operatorname{sign} \operatorname{det} A \neq 0 .
$$




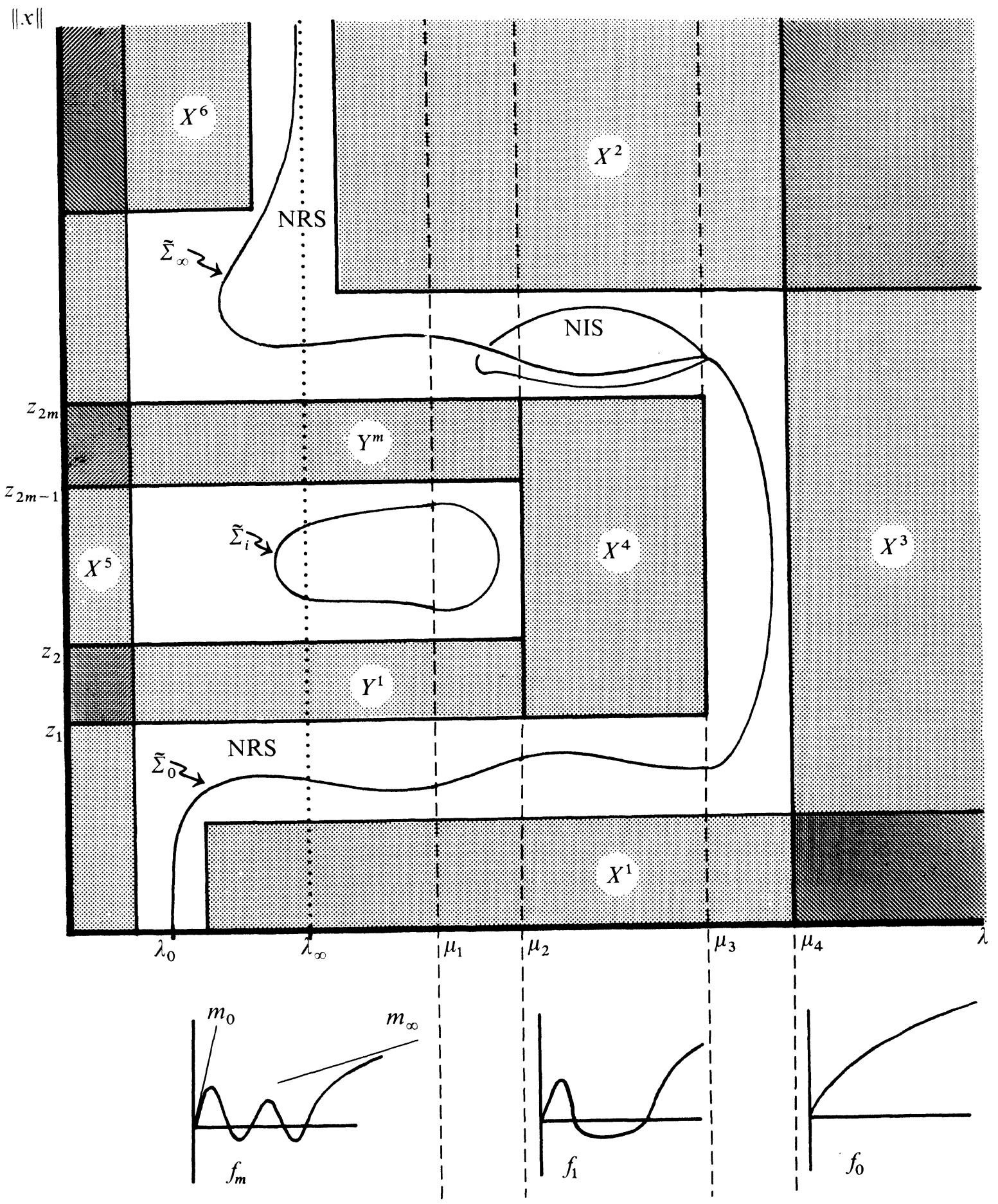

Figure 3

Further the remark following the proof of Theorem 3.2 implies that for $\lambda$ sufficiently large and $\varepsilon>0$ small

$$
\operatorname{deg}\left(\frac{1}{\lambda} A-F_{m}, B(\varepsilon), 0\right)=0 .
$$


Thus

$$
\operatorname{deg}\left(A-\lambda F_{m}, B(\varepsilon), 0\right)_{\lambda<\lambda_{0}} \neq \operatorname{deg}\left(A-\lambda F_{m}, B(\varepsilon), 0\right)_{\lambda>\lambda_{0}}
$$

and hence bifurcation from 0 must occur at $\lambda_{0}$.

Due to the Rabinowitz bifurcation result [12] an unbounded continuum $\tilde{\Sigma}_{0}$ must bifurcate from $\left(\lambda_{0}, 0\right)$. Considering now the regions constructed in (3.9)-(3.15) and since $\delta_{\infty}^{+}$and $\delta_{\infty}^{-}$may be chosen arbitrarily small $\tilde{\Sigma}_{0}$ must become unbounded with $\lambda \rightarrow \lambda_{\infty}$ and $\|x\| \rightarrow \infty$, thus in view of Lemma 2.11 and Remark 2.12, $\lambda_{\infty}$ is the only bifurcation point from infinity.

We now pass to the remaining assertions of Theorem 3.3. It follows from Remark 2. 4 and Corollary 2. 5 that $A x=\lambda F_{m}(x)$ has a unique solution $x_{i}(\lambda)$ near $w_{i}$, for all large $\lambda$ where $\left\{w_{i}\right\} \subseteq \mathbb{R}_{+}^{n}$ are those zeros of $F_{m}$ whose norms are in $\left(z_{1}, z_{2 m}\right)$, whose local degrees are odd. Thus since there are no solutions in the shells $Y^{i}$, $1 \leqq i \leqq m$, either there are no zeros of $\Phi$ in $\overline{B\left(z_{2 j+1}\right)} \backslash B\left(z_{2 j}\right), 1 \leqq j \leqq m-1$ or they generically occur in pairs for $\lambda \leqq \mu_{1}$, simply because $\operatorname{deg}\left(\Phi(\lambda, \cdot), B\left(z_{2 j+1}\right) \backslash \overline{B\left(z_{2 j}\right)}, 0\right)=0$ for $\lambda \leqq \varepsilon$. Since also there are no solutions of (3.8) in $X^{4}$, the continua $\tilde{\Sigma}_{j}$ will reside in $\left[\varepsilon, \mu_{2}\right] \times \overline{B\left(z_{2 j+1}\right)} \backslash B\left(z_{2 j}\right)$, if they exist; therefore they are bounded continua which are disjoint from $\tilde{\Sigma}_{0}$. Moreover, one may show (by means of Whyburn's Lemma (see e.g., [12]) and the fact that they have nonzero local degree) that the continua must be generically homeomorphic to $S^{1}$.

Remark 3. 4. Theorem 3.1 is an immediate consequence of the a priori bounds established in the previous section and the considerations and conclusions of Theorem 3. 3 .

Our proof of Theorem 3. 3 may also be adapted to the situations considered by Ambrosetti and Hess [3] concerning the existence of positive solutions of nonlinear elliptic boundary value problems. Our proof, however, does not need any degree calculations in a neighborhood of $\infty$. Bifurcation from 0 together with the properties of the global perturbation $\Phi$ yields the bifurcation from $\infty$.

We once more remark that superlinear problems may be handled in much the same way where $\lambda_{\infty}=0$ will be the bifurcation point from $\infty$.

We close this section by considering several examples.

Consider the boundary value problem (1.3) where $f=f_{m} \in \mathscr{F}$. The differential equation $u^{\prime \prime}+\mu f(u)=0$ is equivalent to

$$
u^{\prime}=v, v^{\prime}=-\mu f(u),
$$

thus trajectories starting at $\left(0, v_{0}\right)$ of $(3.16)$ must satisfy

$$
\frac{1}{2} v^{2}+\mu \int_{0}^{u} f(s) d s=\frac{1}{2} v_{0}^{2} .
$$

Pick $v_{0}>0$ such that $\left(v_{0}=v_{0}(\mu)\right)$

$$
\frac{1}{2} v_{0}^{2}=\mu \int_{0}^{z_{1}} f(s) d s .
$$


Let us now assume the following:

$$
\text { If } v_{*}>v_{0} \text { and } \frac{1}{2} v_{*}^{2}=\mu \int_{0}^{u} f(s) d s, \text { then } u \geqq z_{2 m} .
$$

In this case the phase portrait of (3.19) will be as follows

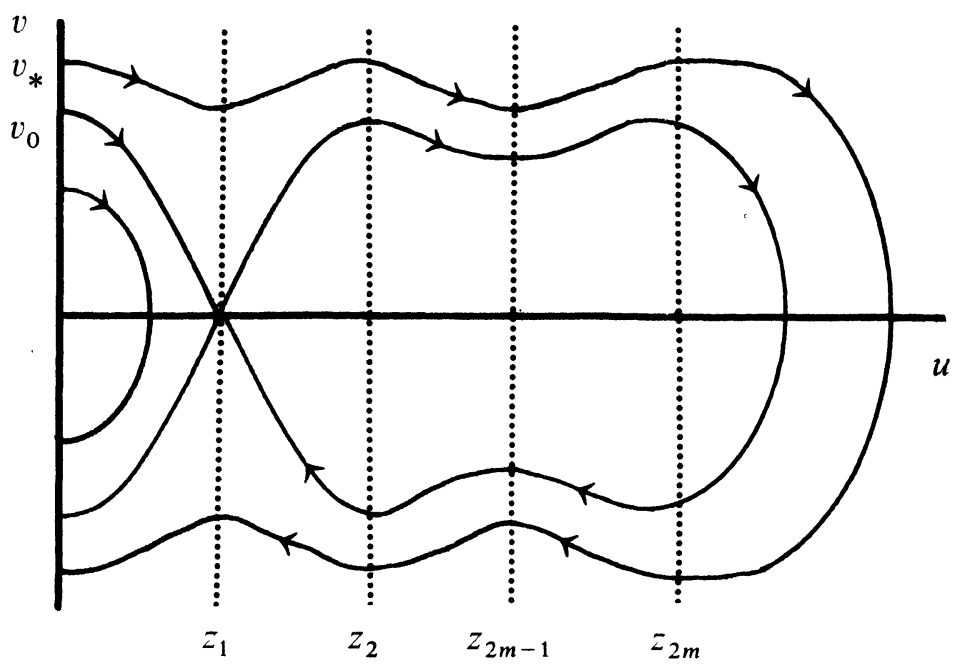

Figure 4

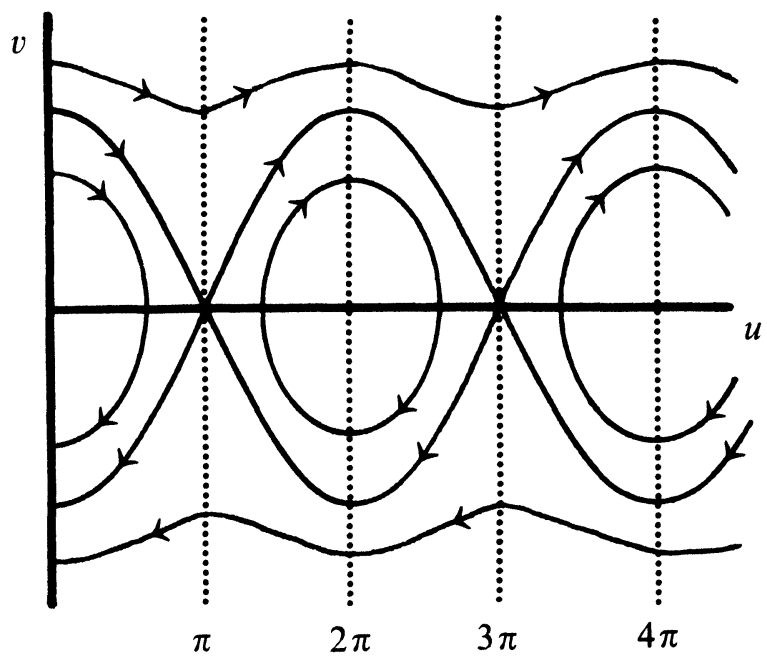

Figure 5

and thus if $u$ is a positive solution of (1.3) it must satisfy either $\|u\|<z_{1}$ or else $\|u\|>z_{2 m}$. In this case all the continua in $B\left(z_{2 i+1}\right) \backslash \overline{B\left(z_{2 i}\right)}, 1 \leqq i \leqq m-1$ must therefore be NIS. It is clear from these considerations that by placing various hypotheses on the behavior of $\int_{0}^{u} f(s) d s$ one may obtain any of $z_{2 k}$ as a possible lower bound on the 
norm of a second possible positive solution and thus, if in (3. 21), $m$ is replaced by $k$ we would obtain NIS in $B\left(z_{2 i+1}\right) \backslash \overline{B\left(z_{2 i}\right)}, 1 \leqq i \leqq k-1$. Further variations of these ideas are of course possible.

If we consider the pendulum equation, then the phase portrait in Figure 5 shows that the only possible positive solution $u$ must satisfy $\|u\|<\pi$. Thus all solutions $(\lambda, x),\|x\|>\pi$, obtained in this case must be NIS. If we consider the conditions of Brown and Budin [5], then some of the $\Sigma_{i}$ may be NRS. In their case [5] the phase portrait is as follows:

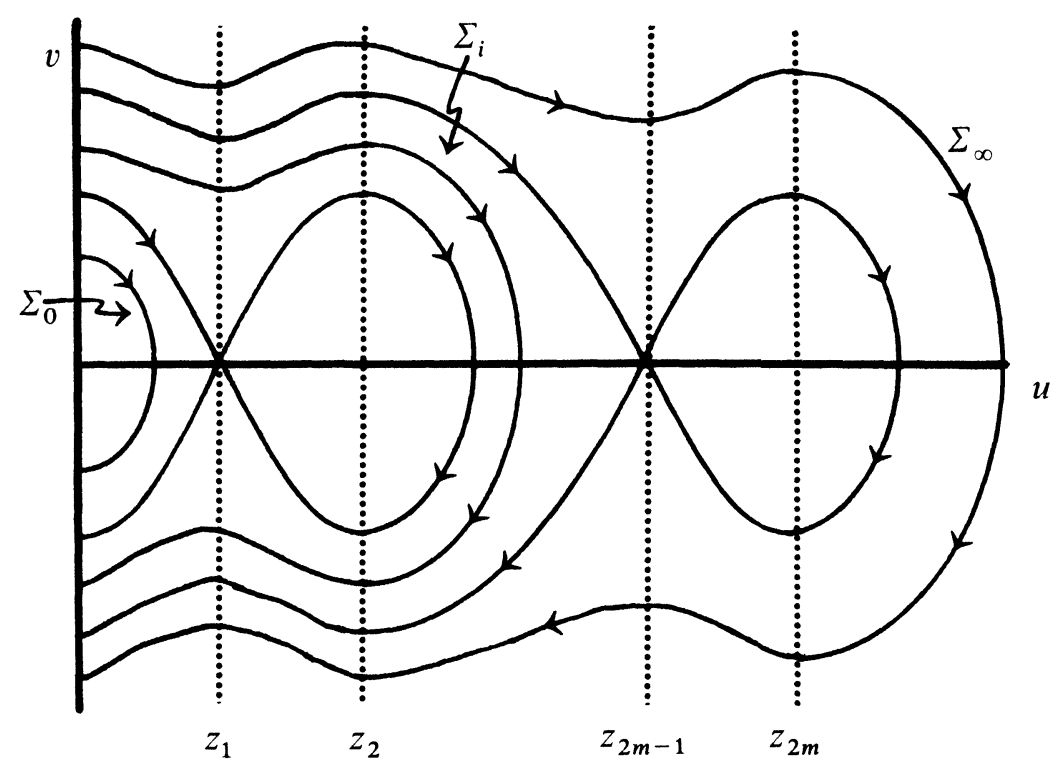

Figure 6

Thus there may be points $v_{1}, v_{2}, \ldots$ (in fact this result is proved in [5] under suitable hypotheses) yielding solutions $u$ of (1.3) with $z_{2}<\|u\|<z_{3}, z_{4}<\|u\|<z_{5}$, etc. These considerations show that the pendulum equation is in some sense on the border line between equations whose phase portrait is as in Figure 4 and those whose phase portrait is as in Figure 6.

\section{Superlinear boundary value problems}

Let $f: \mathbb{R} \rightarrow \mathbb{R}$ be continuous. Some of the following conditions will be assumed at times.

$$
\begin{gathered}
f(x) x>0, x \neq 0, f(x)=o(x) \quad \text { as } x \rightarrow 0 \\
\frac{f(x)}{x} \rightarrow \infty \quad \text { as } x \rightarrow \pm \infty \\
f(x)>0, x \in \mathbb{R} \backslash\{0\}, f(x)=o(x) \quad \text { as } x \rightarrow 0 .
\end{gathered}
$$

Let us, as before, denote by $F$ the $n$-dimensional Nemitskii operator associated with $f$ and let $A$ be an $n \times n$ matrix. We again consider the nonlinear eigenvalue problem

$$
A x=\lambda F(x) .
$$


The motivation for studying this equation is again a class of nonlinear boundary value problems whose solutions are to be obtained via finite difference approximation schemes, a prototype of such problems is given by

$$
u^{\prime \prime}+\lambda u^{m}=0, u(0)=0=u(\pi)
$$

where $\lambda \in \mathbb{R}_{+}$and $m>1$ is an integer.

In studying nontrivial solutions of (4. 5) it suffices to study positive solutions. This is apparent in case $m$ is even. On the other hand, if $m$ is odd then solutions having equally spaced zeros may be pieced together using positive solutions or their negatives. This only involves a change in scale and a uniqueness argument.

The existence results to be given below will not only give nontrivial solution continua but also give via the numerical algorithms of [9] and [11] access to such continua from the trivial solution.

As in the previous chapter we shall see that even though the nonlinear boundary value problem may have a unique positive solution no such uniqueness prevails for the associated equation (4.4) which in fact will have NIS, as will be shown below. turbations.

We first establish a general existence theorem covering two classes of such per-

Let $0<\mu_{1}<\mu_{2}$ be fixed positive numbers and for $\mu_{1} \leqq \lambda \leqq \mu_{2}$ define

$$
H(\lambda, x)=\frac{\lambda-\mu_{2}}{\mu_{1}-\mu_{2}}\left(A x-\mu_{1} F(x)\right)-\frac{\lambda-\mu_{1}}{\mu_{2}-\mu_{1}} x
$$

and define $\Phi: \mathbb{R} \times \mathbb{R}^{n} \rightarrow \mathbb{R}^{n}$ by

$$
\Phi(\lambda, x)= \begin{cases}A x-\lambda F(x), & 0 \leqq \lambda \leqq \mu_{1}, \\ H(\lambda, x), & \mu_{1} \leqq \lambda \leqq \mu_{2}, \\ -x, & \mu_{2} \leqq \lambda .\end{cases}
$$

Thus once $\Phi^{-1}(0)$ is characterized, we will be able to describe the solution set of (4. 4) for $0 \leqq \lambda \leqq \mu_{1}$. Since $\mu_{1}, \mu_{2}$ are arbitrary we will be able to describe the whole solution set of (4. 4).

Theorem 4. 1. (a) Let $A$ be such that

$$
\text { sign } \operatorname{det} A=(-1)^{n+1}
$$

and let $f$ satisfy (4. 1) and (4. 2), or let

(b) $A^{-1}\left(\mathbb{R}_{+}^{n}\right) \subseteq \mathbb{R}_{+}^{n}$ and let $f$ satisfy (4.2) and (4.3) (A nonsingular).

Then there exists $\lambda_{*} \in\left(\mu_{1}, u_{2}\right)$ and an unbounded continuum $\Sigma^{*}$ in $\Phi^{-1}(0)$ which bifurcates from $\left(\lambda_{*}, 0\right)$ such that

$$
\Sigma^{*} \cap\left\{\mu_{1}\right\} \times\left(\mathbb{R}^{n} \backslash\{0\}\right) \neq \emptyset .
$$

In case (b) holds it is true that $\Phi^{-1}(0) \subset \mathbb{R} \times \mathbb{R}_{+}^{n}$ and in fact (4.9) reads

$$
\Sigma^{*} \cap\left\{\mu_{1}\right\} \times\left(\mathbb{R}_{+}^{n} \backslash\{0\}\right) \neq \emptyset .
$$


Proof. We first consider case (a).

There exists $R>0$ such that $(\lambda, x) \in \Phi^{-1}(0)$ and $\mu_{1} \leqq \lambda \leqq \mu_{2}$ implies $\|x\|<R$.

To see this let $H(\lambda, x)=0$, then

$$
A x=\mu_{1} F(x)+\frac{\lambda-\mu_{1}}{\mu_{2}-\lambda} x, \lambda \in\left[\mu_{1}, \mu_{2}\right) .
$$

Thus

$$
\left\|\mu_{1} \frac{F(x)}{\|x\|}+\frac{\lambda-\mu_{1}}{\mu_{2}-\lambda} \frac{x}{\|x\|}\right\| \leqq\|A\|
$$

and hence

$$
\frac{1}{\|x\|}\left|\mu_{1} f\left(x_{i}\right)+\frac{\lambda-\mu_{1}}{\mu_{2}-\lambda} x_{i}\right| \leqq\|A\|, 1 \leqq i \leqq n
$$

since $f$ satisfies (4. 1) it follows that

$$
\begin{gathered}
\mu_{1}\left|\frac{f\left(x_{i}\right)}{\|x\|}\right| \leqq\|A\|, \\
\frac{\lambda-\mu_{1}}{\mu_{2}-\lambda}\left|\frac{x_{i}}{\|x\|}\right| \leqq\|A\|, 1 \leqq i \leqq n .
\end{gathered}
$$

Since $f$ satisfies (4.2) and $\|x\|=\left|x_{j}\right|$ for some $j$, we obtain a bound on $\|x\|$ from

(4. 12). (4. 13) implies

$$
\lambda \leqq \frac{\mu_{2}\|A\|+\mu_{1}}{\|A\|+1}=\bar{\mu}<\mu_{2} .
$$

Since $A$ is nonsingular it follows that 0 is an isolated zero of $\Phi(\lambda, x)$ for $\lambda=\mu_{1}, \mu_{2}$ and we may compute for all small $\delta>0$

$$
\begin{gathered}
i_{\mu_{1}}=\operatorname{deg}\left(\Phi\left(\mu_{1}, \cdot\right), B(\delta), 0\right)=\operatorname{sign} \operatorname{det} A=(-1)^{n+1}, \\
i_{\mu_{2}}=\operatorname{deg}\left(\Phi\left(\mu_{2}, \cdot\right), B(\delta), 0\right)=\operatorname{deg}(-\operatorname{id}, B(R), 0)=(-1)^{n} .
\end{gathered}
$$

Hence since $i_{\mu_{1}} \neq i_{\mu_{2}}, \Phi^{-1}(0)$ must undergo bifurcation as $\lambda$ ranges over $\left[\mu_{1}, \mu_{2}\right]$, further $\lambda \leqq \bar{\mu}$ (see (4. 14)). It follows also that

$$
\operatorname{deg}\left(\Phi\left(\mu_{1}, \cdot\right), B(R) \backslash \overline{B(\delta)}, 0\right)=(-1)^{n}-(-1)^{n+1}=2(-1)^{n},
$$

hence letting

$$
\mathscr{A}=\Phi^{-1}(0) \cap\left\{\mu_{1}\right\} \times B(R) \backslash \overline{B(\delta)} \neq \emptyset \text { and } \mathscr{B}=\left[\mu_{1}, \mu_{2}\right] \times\{0\},
$$

$\mathscr{A}$ and $\mathscr{B}$ must be connected in $\Phi^{-1}(0)$ due to Whyburn's lemma and due to the homotopy property of Brouwer degree there will be a continuum $\Sigma^{*}$ bifurcating from $\left(\mu_{1}, \mu_{2}\right) \times\{0\}$ which hits $\left\{\mu_{1}\right\} \times(B(R) \backslash \overline{B(\delta)})$.

Case (b). Since $F: \mathbb{R}^{n} \rightarrow \mathbb{R}_{+}^{n}$ and $A^{-1}$ is positive we consider equation (4.4) in $\mathbb{R}_{+}^{n}$ and use fixed point index rather than degree theory. 
Again if $(\lambda, x)$ is a nontrivial solution of (4. 4), $x \in \mathbb{R}_{+}^{n}, \mu_{1} \leqq \lambda \leqq \mu_{2}$, then (4. 11) holds and since $\|\cdot\|$ is monotone with respect to the ordering induced by $\mathbb{R}_{+}^{n}$ it follows from (4. 11) that (4.14) holds and $\mu_{1}\left\|\frac{F(x)}{\|x\|}\right\| \leqq\|A\|$. Thus again there exists $R>0$ such that $(\lambda, x) \in \Phi^{-1}(0), \mu_{1} \leqq \lambda \leqq \bar{\mu}$, implies $\|x\|<R$. On the other hand $(\lambda, x) \in \Phi^{-1}(0)$, $\mu_{1} \leqq \lambda \leqq \bar{\mu}$ if and only if $x$ is a fixed point of $\Psi(\lambda, x)$, where

$$
\Psi(\lambda, x)=\mu_{1} A^{-1} F(x)+\frac{\lambda-\mu_{1}}{\mu_{2}-\lambda} A^{-1} x .
$$

We now compute the fixed point index of $\Psi(\lambda, \cdot)$ at 0 for $\lambda=\mu_{1}$ and for $\lambda$ close to $\mu_{2}$. Since $A^{-1}(F(x))=o(\|x\|)$ as $x \rightarrow 0$ it follows that the fixed point index of $\psi$ at 0 for $\lambda=\mu_{1}$, ind $\left(\mathbb{R}_{+}^{n}, \Psi\left(\mu_{1}, \cdot\right), B(\delta)\right)=1$.

We next show that for $\lambda$ sufficiently close to $\mu_{2}$ the index equals 0 .

To see this let $\tilde{\Psi}$ be a Dugundji extension of $\Psi$ to $\mathbb{R}^{n+1}$ (i.e., $\tilde{\Psi}\left(\mathbb{R}^{n+1}\right) \subseteq \mathbb{R}_{+}^{n}$ ), then ind $\left(R_{+}^{n}, \Psi(\lambda, \cdot), B(\delta)\right)=\operatorname{deg}($ id $-\tilde{\Psi}(\lambda, \cdot), B(\delta), 0)=\operatorname{deg}($ id $-\tilde{\Psi}(\lambda, \cdot), B(\delta), \bar{\varepsilon})$

for $\|\bar{\varepsilon}\|$ small. Thus, it suffices to show that

$$
x-\tilde{\Psi}(\lambda, x)=\bar{\varepsilon}
$$

has no solutions for $\bar{\varepsilon} \in \mathbb{R}_{+}^{n} \backslash\{0\}$ of small norm. If $x$ is such a solution, then since $x=\bar{\varepsilon}+\widetilde{\Psi}(\lambda, x), x \in \mathbb{R}_{+}^{n}$. Proceeding indirectly, we find sequences $\left\{\varepsilon_{m}\right\} \leqq \mathbb{R}_{+}^{n} \backslash\{0\}, \varepsilon_{m} \rightarrow 0$ and $\left\{x_{m}\right\} \subseteq \mathbb{R}_{+}^{n}$ and $\left\{\lambda_{m}\right\}, \lambda_{m} \rightarrow \mu_{2}$ such that

$$
x_{m}=\Psi\left(\lambda, x_{m}\right)+\varepsilon_{m},
$$

hence

$$
\left(\mu_{2}-\lambda_{m}\right) x_{m}=\mu_{1}\left(\mu_{2}-\lambda_{m}\right) A^{-1} F\left(x_{m}\right)+\left(\lambda_{m}-\mu_{1}\right) A^{-1} x_{m}+\left(\mu_{2}-\lambda_{m}\right) \varepsilon_{m} .
$$

We consider three cases.

Suppose $\left\|x_{m}\right\| \rightarrow \infty$. We divide both sides of (4.16) by $\left\|x_{m}\right\|$ and obtain

$$
\left(\lambda_{m}-\mu_{1}\right) A^{-1} \frac{x_{m}}{\left\|x_{m}\right\|}=-\mu_{1}\left(\mu_{2}-\lambda_{m}\right) A^{-1} \frac{F\left(x_{m}\right)}{\left\|x_{m}\right\|}+\delta_{m}
$$

where $\delta_{m} \rightarrow 0$. Since $\frac{F\left(x_{m}\right)}{\left\|x_{m}\right\|} \in \mathbb{R}_{+}^{n}$ and $A^{-1}$ is bounded we obtain a contradiction.

Suppose $\left\|x_{m}\right\| \rightarrow 0$. We again divide (4. 16) by $\left\|x_{m}\right\|$ and obtain

$$
\left(\lambda_{m}-\mu_{1}\right) A^{-1} \frac{x_{m}}{\left\|x_{m}\right\|}+\frac{\mu_{2}-\lambda_{m}}{\left\|x_{m}\right\|} \varepsilon_{m}=\delta_{m},
$$

where $\delta_{m} \rightarrow 0$. We thus obtain a contradiction since $\left(\lambda_{m}-\mu_{1}\right) A^{-1} \frac{x_{m}}{\left\|x_{m}\right\|}$ has a subsequence converging to $y \in \mathbb{R}_{+}^{n} \backslash\{0\}$ and $\frac{\mu_{2}-\lambda_{m}}{\left\|x_{m}\right\|} \varepsilon_{m} \in \mathbb{R}_{+}^{n}$. The case that $\left\|x_{m}\right\|$ is bounded away from 0 and infinity is argued in much the same way. 
Thus the fixed point index of $\Psi(\lambda, \cdot)$ at 0 equals 0 for $\lambda$ close to $\mu_{2}$. An argument similar to the one used in case (a) thus leads to the conclusion that a continuum of nontrivial solutions will bifurcate from $\left(\mu_{1}, \mu_{2}\right)$ which is contained in $\mathbb{R}_{+}^{n}$ connecting to $\left\{\mu_{1}\right\} \times B(R) \backslash \overline{B(\delta)}$. This continuum will now reside in $\left[\mu_{1}, \mu_{2}\right] \times \mathbb{R}_{+}^{n}$.

Remark 4. 2. Since in both cases of Theorem 4.1 we may in fact assert that an unbounded continuum will bifurcate and since solutions are a priori bounded for $\lambda \in\left[\mu_{1}, \mu_{2}\right]$ it in fact follows that the continuum $\Sigma^{*}$ will become unbounded for $\lambda \in\left(0, \mu_{1}\right)$ and since $A$ is nonsingular it follows that if $\left(\lambda, x_{\lambda}\right) \in \Sigma^{*}$ with $|\lambda|+\left\|x_{\lambda}\right\| \rightarrow \infty$ then it must be the case that $\lambda \rightarrow 0+$. It thus also follows that the original problem has an unbounded continuum of nontrivial solutions for $0<\lambda \leqq \mu_{1}$ which may be obtained via a continuation technique as a bifurcating continuum for the global perturbation $\Phi$.

$$
A x-\lambda F(x)=0 \quad H(x, \lambda)=0 \quad-\operatorname{Id}(x)=0
$$

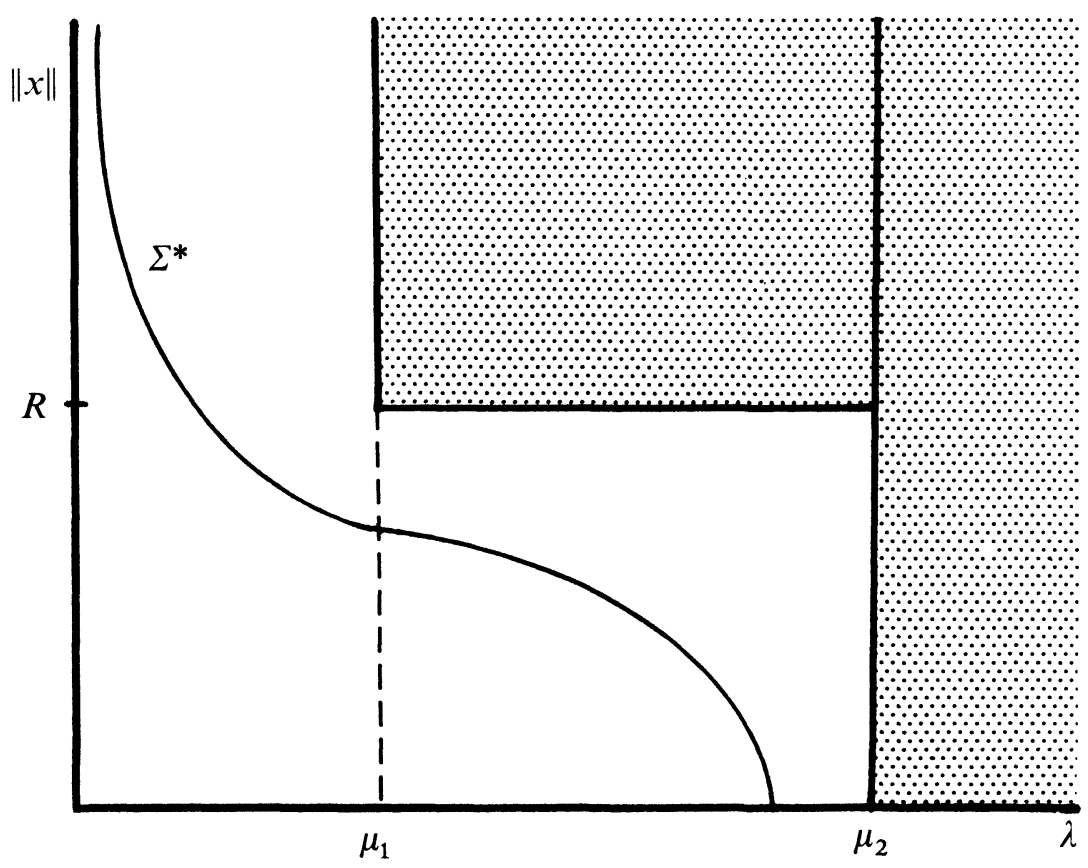

Figure 7

Remark 4. 3. If instead of the approach used in Theorem 4. 1 one establishes a homotopy to a constant mapping the necessary a priori bounds and index calculations are much easier to obtain, however this type of homotopy argument, even though leading to solutions of our problem, may lead to NIS and hence is not appropriate. This is demonstrated in Figures 27 and 28.

In the case $f(x)=x^{k}$ we shall show that the global perturbation of Theorem 4. 1 indeed will lead to NRS avoiding the NIS. It is this specific case which will be considered now, i.e., we assume

$$
f(x)=x^{k}, k>1 \text { an integer. }
$$


Equation (4. 4) thus is considered to be a finite difference approximation to the problem

$$
u^{\prime \prime}+\mu u^{k}=0, u(0)=0=u(\pi),
$$

i.e., $A$ is the discretization of the differential operator $-u^{\prime \prime}$ with boundary condition $u(0)=0=u(\pi)$ on a uniform grid of $n$ internal meshpoints, i.e., $A$ is given as in section 3 .

The following lemma, which is due to Allgower [1], will be of essential use in our discussion.

Lemma 4. 4. Let $A$ and $f$ be as above. Then:

(i) For every $\lambda>0$ there exists a unique solution $x \in \mathbb{R}_{+}^{n}$ of (4. 4) which is symmetric about $\frac{\pi}{2}$ and this solution is an approximation to the unique positive solution of (4.19) $\left(\lambda=h^{2} \mu, h\right.$ the meshsize $)$.

(ii) Equation (4.4) admits NIS which are characterized by the fact that they are not symmetric about $\frac{\pi}{2}$ and their existence is independent of $\lambda$.

Remark 4. 5. The NIS for the present problem are different from those of the problems considered earlier in the sense that their existence is independent of $\lambda$ and that for a fixed $\lambda$ they disappear as the meshsize tends to 0 , whereas those considered earlier are present for each meshsize but then only for large $\lambda$.

In order to obtain nontrivial positive solutions of (4.4) we shall show that the perturbations introduced in Theorem 4. 1 yield symmetric (about $\left.\frac{\pi}{2}\right)$ NRS.

In view of this define the space $X \subset \mathbb{R}^{n}$ by

$$
X=\left\{x \in \mathbb{R}^{n}: x_{i}=x_{n-i+1}, i=1, \ldots,\left[\frac{n}{2}\right]\right\}
$$

and identify elements $x=\left(x_{1}, \ldots, x_{n}\right) \in X$ with $x=\left(x_{1}, \ldots, x_{m}\right)$, where $m=\frac{n}{2}$ if $n$ is even and $m=\left[\frac{n}{2}\right]+1$ if $n$ is odd. We denote $X \cap \mathbb{R}_{+}^{n}$ by $X_{+}$.

Theorem 4. 6. Define $\Phi$ (in case $k$ is odd) and $\Psi$ (in case $k$ is even) as in Theorem 4. 1. Then:

(i) $\Phi(X) \subseteq X, \Psi\left(X_{+}\right) \subseteq X_{+}$.

(ii) There exists a unique continuum $\Sigma_{0}$ of positive solutions of $\Phi(\lambda, x)=0$, respectively $\Psi(\lambda, x)=x$ bifurcating from 0 . Furthermore, $\Sigma_{0}$ leads to positive solutions of (4.4) for $\lambda=\mu_{1}$ which by the choice of $X$ are NRS $\left(\Sigma_{0} \subseteq R_{+} \times X_{+}\right)$.

(iii) In $X$, respectively $X_{+}$, no NIS will occur and in $\mathbb{R}^{n}$, respectively $\mathbb{R}^{n}{ }_{+}$, (positive) NIS occur in pairs. 
Proof. (i) This assertion follows immediately from the definition of $\Phi$ and $\Psi$ and the special structure of $A$.

(ii) $A$ has exactly one positive eigenvalue of multiplicity one corresponding to a strictly positive eigenvector (see Lemma 2.6). Since $\frac{\lambda-\mu_{1}}{\mu_{2}-\lambda}$ ranges over $[0, \infty$ ) as $\lambda$ ranges over $\left[\mu_{1}, \mu_{2}\right], \Phi$ and $\Psi$ will undergo a bifurcation for $\lambda_{=} \lambda_{*} \in\left(\mu_{1}, \mu_{2}\right)$ (see [9] for $\Phi$ and Theorem 4.1 for $\Psi$ ). In either case the bifurcating continuum $\Sigma_{0}$ will be unbounded as follows from the Sturm-Liouville theory for second order difference equations, respectively Theorem 4.1 part (b). Hence $\Sigma_{0}$ will intersect $\left\{\mu_{1}\right\} \times\left(X_{+} \backslash\{0\}\right)$.

(iii) Let

$$
z=\left(z_{1}, \ldots, z_{n}\right)
$$

be a solution of (4.4) which is not symmetric about $\left(\frac{\pi}{2}\right)$, i.e. NIS. Then due to the special structure of $A$ also

$$
\bar{z}=\left(z_{n}, \ldots, z_{1}\right)
$$

is a solution of (4. 4). Moreover, the local degrees (resp. indices) add up to zero.

Remark 4. 7. Theorem (4.6) means that the perturbations $\Phi$ (resp. $\Psi$ ) preserve the symmetry distinguishing NRS from NIS. Thus, $\Sigma_{0}$ guarantees a numerical access to the NRS of problem (4. 4) from trivial solutions.

\section{Numerical results and experience}

All computations were performed by means of an interactive package of $P L$-algorithms which are an implementation and realization of the ideas described in [9] and [11]. These algorithms are designed for a global numerical study of nonlinear eigenvalue and bifurcation problems

$$
H(\lambda, x)=0
$$

where $H: \mathbb{R} \times \mathbb{R}^{n} \rightarrow \mathbb{R}^{n}$ is continuous.

We briefly summarize the basic numerical problems and procedures described in [9] and [11]. The problems are basically the following: (i) To globally follow a given continuum in $H^{-1}(0)$ without being stopped by the presence of cusps, turning and bifurcation points. (ii) To find all bifurcation points and bifurcating branches. (iii) To find all continua in $H^{-1}(0)$.

The procedures to accomplish these tasks are based on a global numerical unfolding

$$
K: R \times R^{n} \times R \rightarrow R^{n}
$$

of $H^{-1}(0)$. More precisely, $H^{-1}(0)$ is replaced in two approximating steps: 
(i) Let $T$ be a triangulation of $R^{n+1}$ and let $H_{T}$ denote the natural $P L$-approximation to $H$ defined by $T$. Then $H^{-1}(0)$ is replaced by $H_{T}^{-1}(0)$. This step corresponds to a mollifying procedure in the $C^{\infty}$-category.

(ii) For $\varepsilon>0$ sufficiently small the problem $H_{T}^{-1}(0)$ is replaced by $H_{T}^{-1}(\bar{\varepsilon})$, where $\bar{\varepsilon}=\left(\varepsilon, \varepsilon^{2}, \ldots, \varepsilon^{n}\right)$. One shows that $\bar{\varepsilon}$ is a regular value in the $P L$ sense and this step corresponds to a regularizing procedure (Brown-Sard) in the $C^{\infty}$-category.

Define

$$
K(\lambda, x, \varepsilon)=H_{T}(\lambda, x)-\bar{\varepsilon} .
$$

Then one can show that $\mathscr{M}(\varepsilon)=K^{-1}(0) \cap R \times R^{n} \times\{\varepsilon\}$ is a collection of $P L$ manifolds of dimension 1 and this unfolding is what we previously have called the generic case in the $P L$ sense. Each of these manifolds in $\mathscr{M}(\varepsilon)$ can be numerically traced by a simplicial algorithm and moreover the global structure and behavior of $\mathscr{M}(\varepsilon)$ can be completely understood through the properties of Brouwer degree. Especially the Leray-Schauder Continuation Method and the Bifurcation Principles of Krasnosel'skii and Rabinowitz may be interpreted in this approach and become numerical tools. This describes to a certain extent the procedure for (i) above. The techniques for (ii) and (iii) are more intricate and quite elaborate, we refer to [9] and [11] for a detailed description. We note, however, that $\Phi$ and $\Psi$ (of section 3 and 4) are typical global perturbations belonging to the category of (ii) and (iii).

All figures in this section are computer plots and represent the $P L$ manifolds in $H_{T}^{-1}(\bar{\varepsilon})$. They have been produced on an Evans and Sutherland picture system.

The following numerical results should be strictly understood to contribute to a global numerical study, i.e., the primary purpose is to exhibit the qualitative nature of $H^{-1}(0)$. Local efficiency and accuracy have been given secondary consideration, and thus at times local accuracy has been sacrificed in order to be able to work with low dimensional difference approximations; our theoretical study, on the other hand, also shows that extreme care must be taken in using solutions of difference equations as approximations to solutions of differential equations. Occurance of NIS demonstrates the necessity of a global study in addition to a local numerical study. We now present several examples.

\section{The asymptotically linear problem (1.3)}

Instead of (1.3) we analyze the problem

$$
u^{\prime \prime}+\mu f(u)=0, u(0)=u^{\prime}(\pi / 2)=0,
$$

since symmetric extensions (about $\pi / 2$ ) of a solution of (5.1) will yield a solution of (1. 3). (5.1) has been discretized on a uniform mesh of $n$ internal meshpoints. To obtain access to solutions for large $\lambda$ we employ the transformation

$$
\lambda=\tilde{\lambda} \exp (\tilde{\lambda}-1)
$$

and solve the problem

$$
A x-\tilde{\lambda} \exp \left(\tilde{\lambda}_{r-1}\right) F(x)=0 .
$$




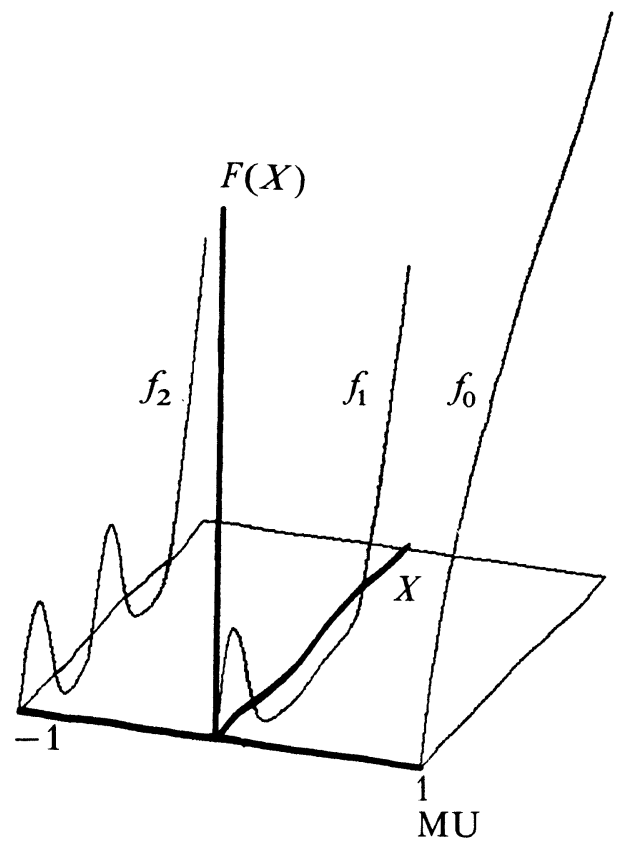

Figure 8

In Figure 8 we show three typical nonlinearities $f_{2}, f_{1}, f_{0}$. Let

$$
\begin{gathered}
\tilde{f_{2}}(x)= \begin{cases}\frac{1}{2} \sin 2 x, & 0 \leqq x \leqq 2 \pi \\
x-2 \pi, & 2 \pi \leqq x \leqq 3 \pi \\
\frac{1}{3} \mathbf{x}+\frac{2}{3} \sin a(x), & 3 \pi \leqq x<\infty\end{cases} \\
\tilde{f_{1}}(x)= \begin{cases}\frac{1}{2} \sin 2 x, & 0 \leqq x \leqq \frac{\pi}{2} \\
-\frac{3}{2} \sin \frac{2}{3}\left(x-\frac{\pi}{2}\right), & \frac{\pi}{2} \leqq x \leqq 2 \pi \\
\tilde{f_{2}}(x), & 2 \pi \leqq x<\infty\end{cases}
\end{gathered}
$$

and

$$
\tilde{f_{0}}(x)=\frac{2}{3} \sin b(x)+\frac{x}{3}, 0 \leqq x<\infty
$$

where

$$
a(x)=\pi(x-3 \pi)[\pi+(x-3 \pi)]^{-1}, b(x)=\pi x[\pi+x]^{-1}
$$

and finally set

$i=0,1,2$.

$$
f_{i}(x)=\left\{\begin{array}{l}
\tilde{f_{i}}(x), \text { if } \tilde{f_{i}}(x) \geqq 0, \\
-\frac{1}{2}\left(1-\exp \left(-2\left|\tilde{f_{i}}(x)\right|\right),\right. \text { otherwise, }
\end{array}\right.
$$


Observe that the $f_{i}$ are chosen as examples for elements of $\mathscr{F}$. The next figure shows the respective continua of solutions.

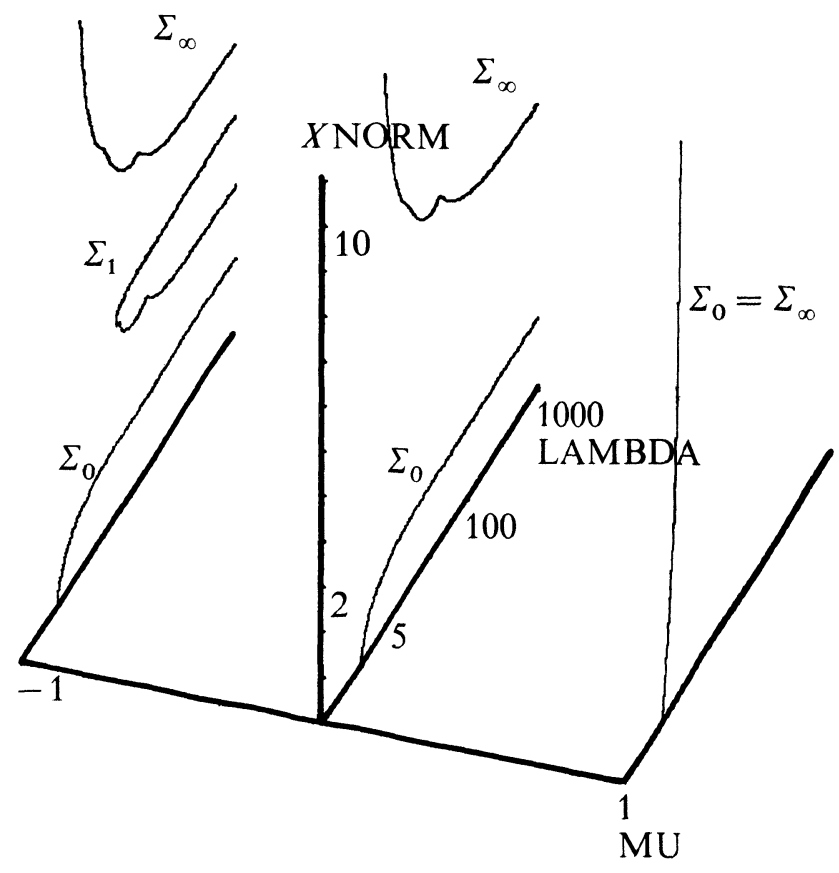

Figure 9

The continua $\Sigma_{0}$ have been computed by means of a bifurcation algorithm in the package. The continua $\Sigma_{1}$ and $\Sigma_{\infty}$ have been obtained by finding $\Psi^{-1}(0)$ for different values of $\tilde{\lambda}$, where

$$
\Psi(\tau, x)=(1-\tau)[A x-\tilde{\lambda} \exp (\tilde{\lambda}-1) F(x)]+\tau d,
$$

and $d$ is the constant $n$-vector, $\operatorname{col}(0,0, \ldots, 0,1)$. The discretization in Figures 9 through 15 has been on $n=6$ equally spaced interval meshpoints. We note that the $\Sigma_{1}$ and $\Sigma_{\infty}$ components show cusps and folds. In Figure 10 we have embedded the three nonlinearities $f_{2}, f_{1}, f_{0}$ into a 1 -parameter family

$$
h(\mu, x)=\left\{\begin{array}{l}
-\mu f_{2}(x)+(\mu+1) f_{1}(x),-1 \leqq \mu \leqq 0 \\
(1-\mu) f_{1}(x)+\mu f_{0}(x), 0 \leqq \mu \leqq 1
\end{array}\right.
$$

Define the two parameter problem

$$
\Phi(\tilde{\lambda}, \mu, x):=A x-\tilde{\lambda} \exp (\tilde{\lambda}-1) H(\mu, x)=0,
$$

where $H$ is the Nemitskii operator associated with $h$. Then $\Phi$ is the realization of the global perturbation discussed in Section 3. Figures 11, 12 and 13 show the solution surfaces from various perspectives.

These results support our theoretical analysis that $\Phi^{-1}(0)$ connects the continua $\Sigma_{0}$ and $\Sigma_{\infty}$ while not intersecting the continuum labelled $\Sigma_{1}$. Hence, if $\Sigma_{1}$ consisted of NIS then the perturbation (5.5) provides a numerical access to the NRS in $\Sigma_{\infty}$ via those in $\Sigma_{0}$ and following the surface for fixed $\tilde{\lambda}$ as $\mu$ varies. 


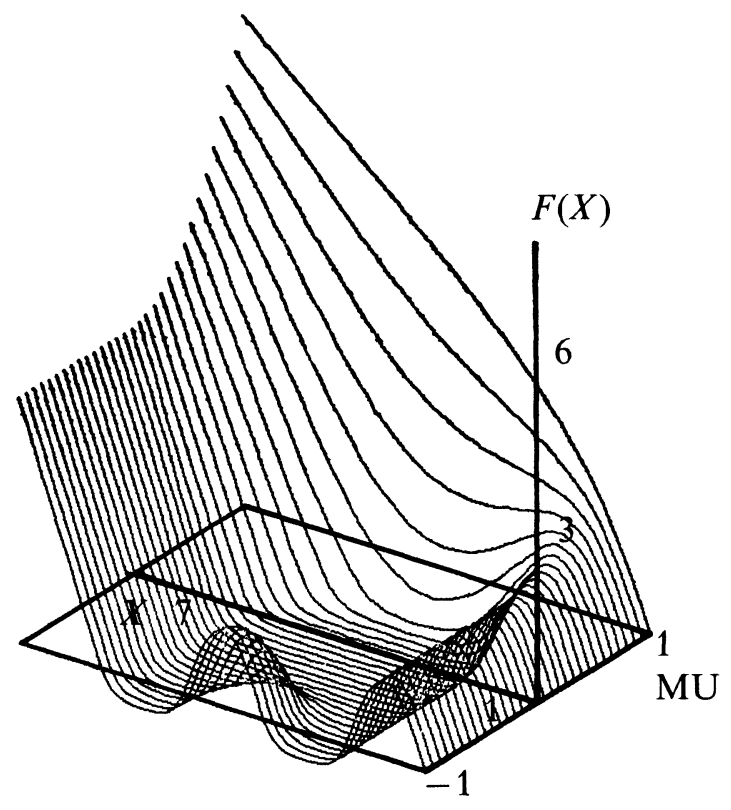

Figure 10

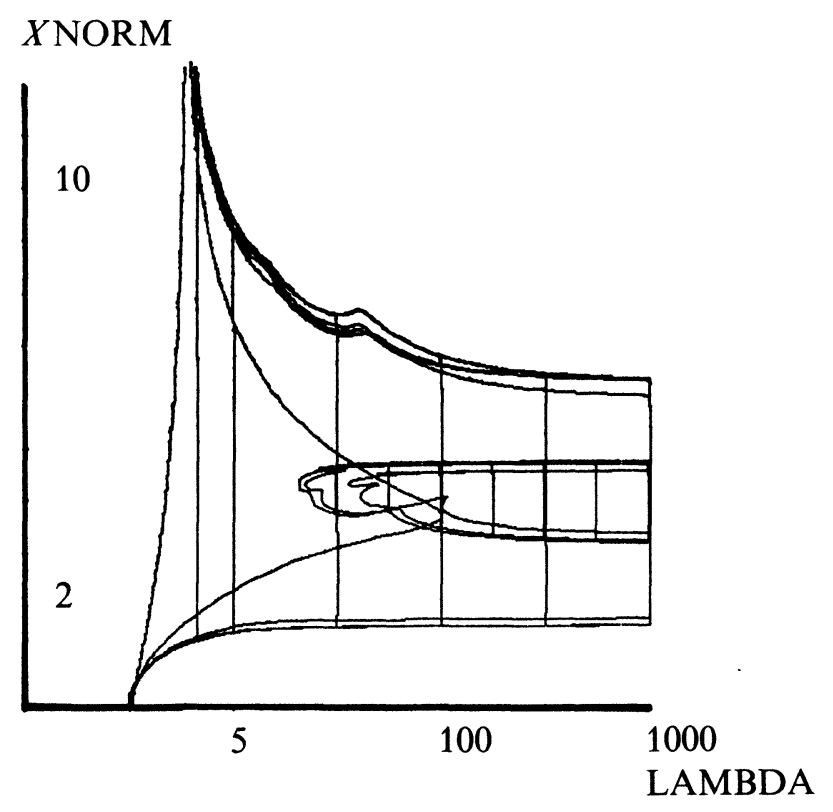

Figure 11

All surfaces show several cusps and folds which are shown in two closeups, Figures 14 and 15.

These results suggest - and this is supported by the studies represented in Figures $11,16,17$ - that these phenomena are due to singularities in $\Phi^{-1}(0)$ and not - as one might surmise - due to numerical imperfections. 


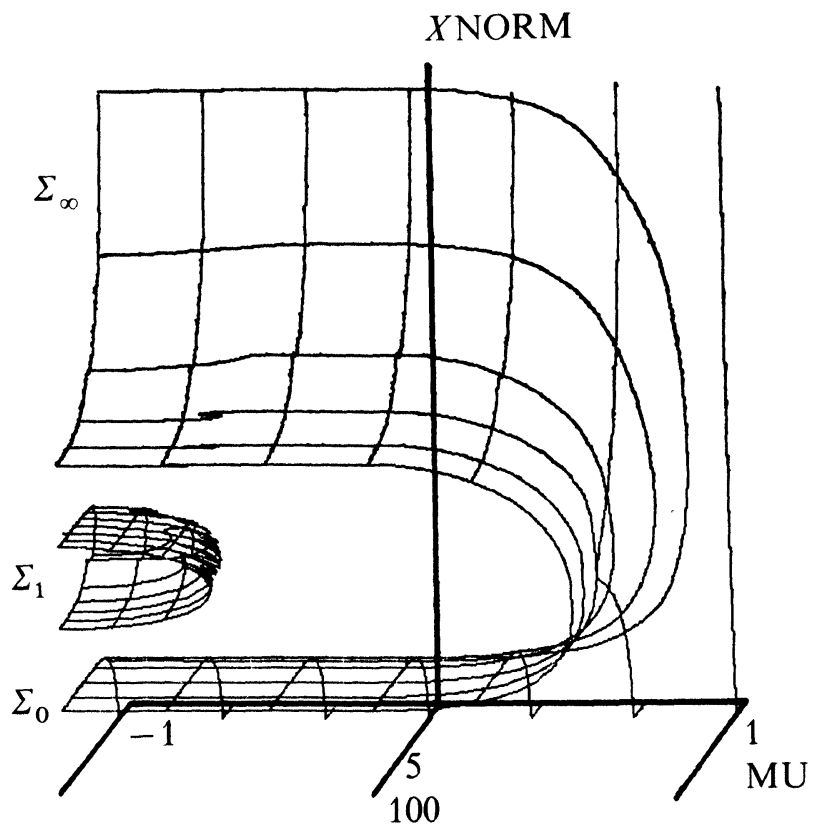

1000

LAMBDA

Figure 12

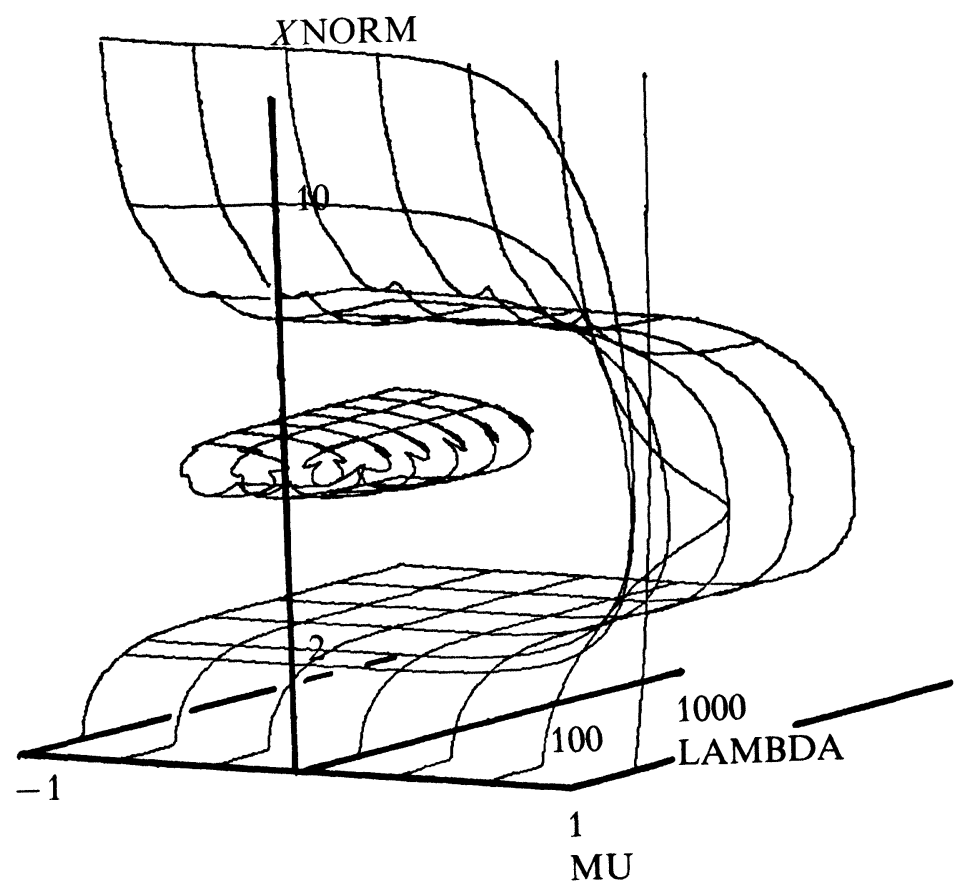

Figure 13 


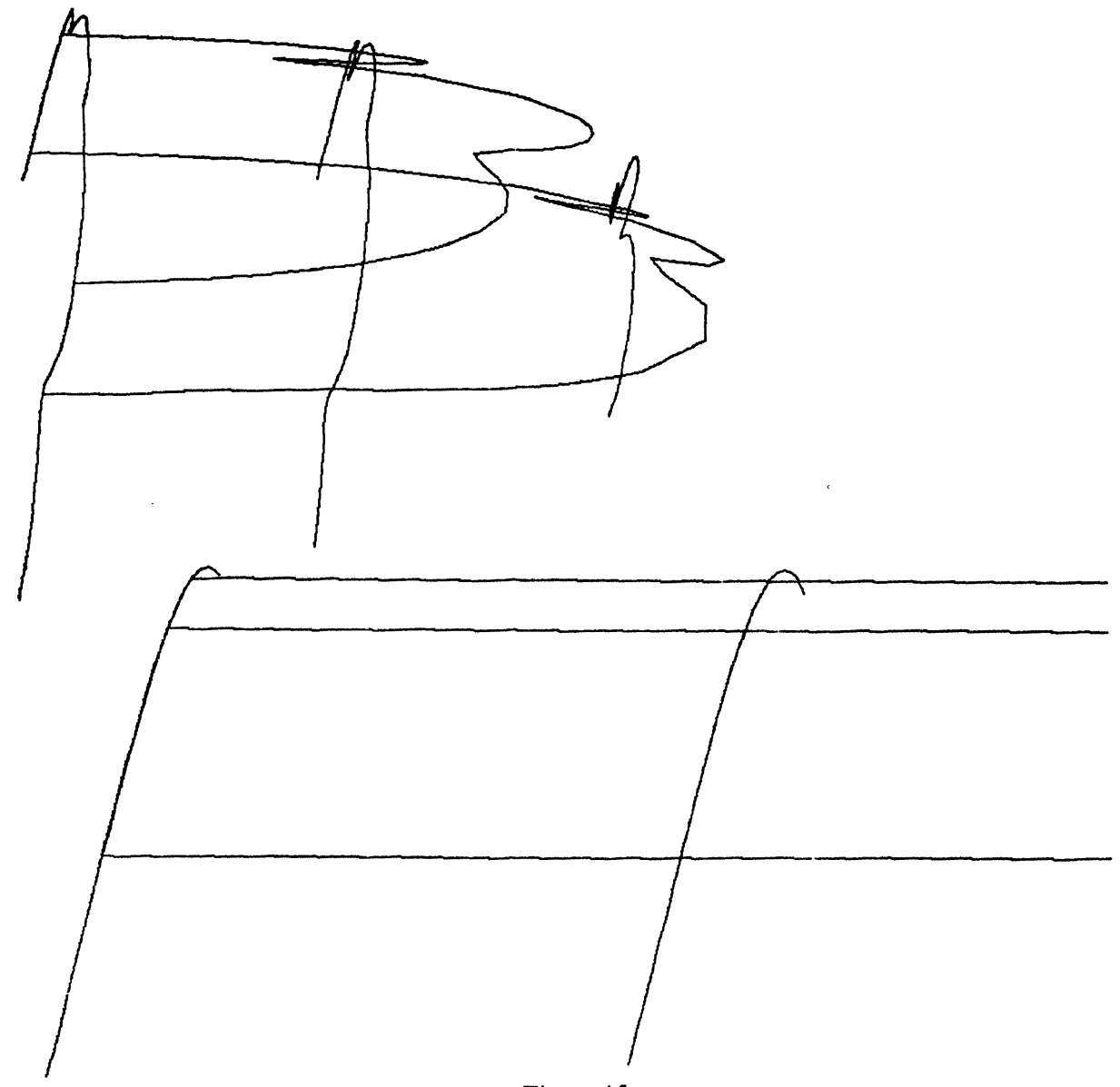

Figure 15

Figure 16 and 17 show the dependence of the "cusp" in $\Sigma_{\infty}$ upon the meshsize of the discretization of (5.1). It suggests itself that the "cusp" in Figure 16 appears for all meshsizes, but moves to infinity with the meshsize tending to zero. Hence the "cusp" seems typical for the algebraic system (5. 3). Projecting Figure 16 onto the $(\lambda,\|x\|)$ plane, as given in Figure 17, shows that the continua $\Sigma_{0}$ are quite stable with varying meshsize in contrast to the continua $\Sigma_{\infty}$. Figures 18 to 26 are solution plots of another typical example for (1.3).

The nonlinearity is given as

$$
f_{2}(x)= \begin{cases}\frac{1}{2} \sin 2 x, & 0 \leqq x \leqq 2 \pi \\ x-2 \pi, & 2 \pi \leqq x \leqq \infty\end{cases}
$$

According to Figure 5 and the remarks following it, the boundary value problem (5. 1) with this nonlinearity cannot have positive solutions such that $\frac{\pi}{2} \leqq\|u\| \leqq 2 \pi$. However, Theorem 3. 2 implies that (5. 3) must have at least $4^{6}=4096$ nontrivial solutions $x$ in $\mathbb{R}_{+}^{6}$ of which there are at least 728 with norm $\pi \leqq\|x\| \leqq \frac{3}{2} \pi$ and 3367 with norm $\|x\| \geqq 2 \pi$, where we again have chosen 6 internal meshpoints. Figure 18 shows 10 NIS and the NRS components $\Sigma_{0}$ and $\Sigma_{\infty}$. 
106
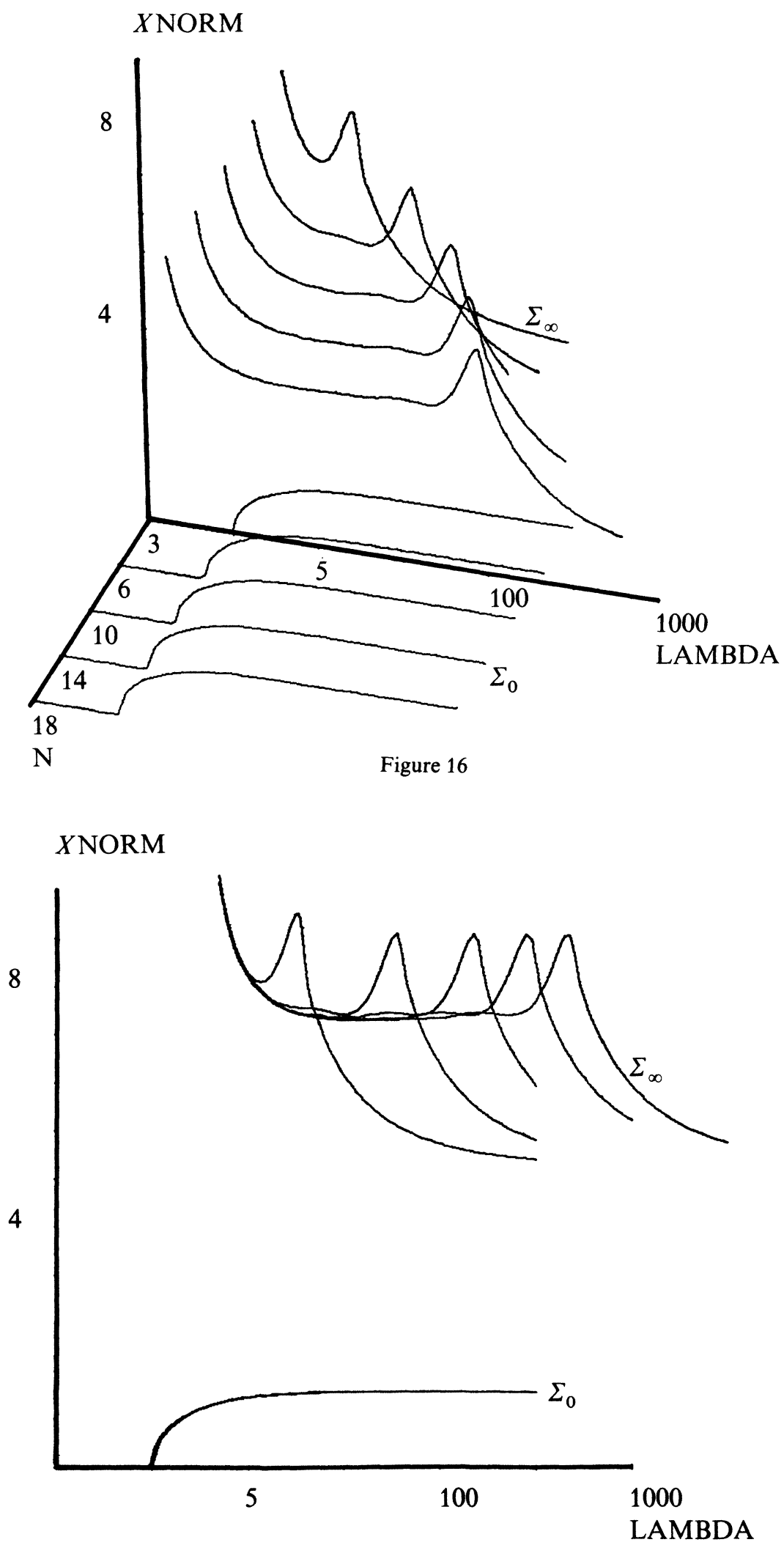

Figure 17 


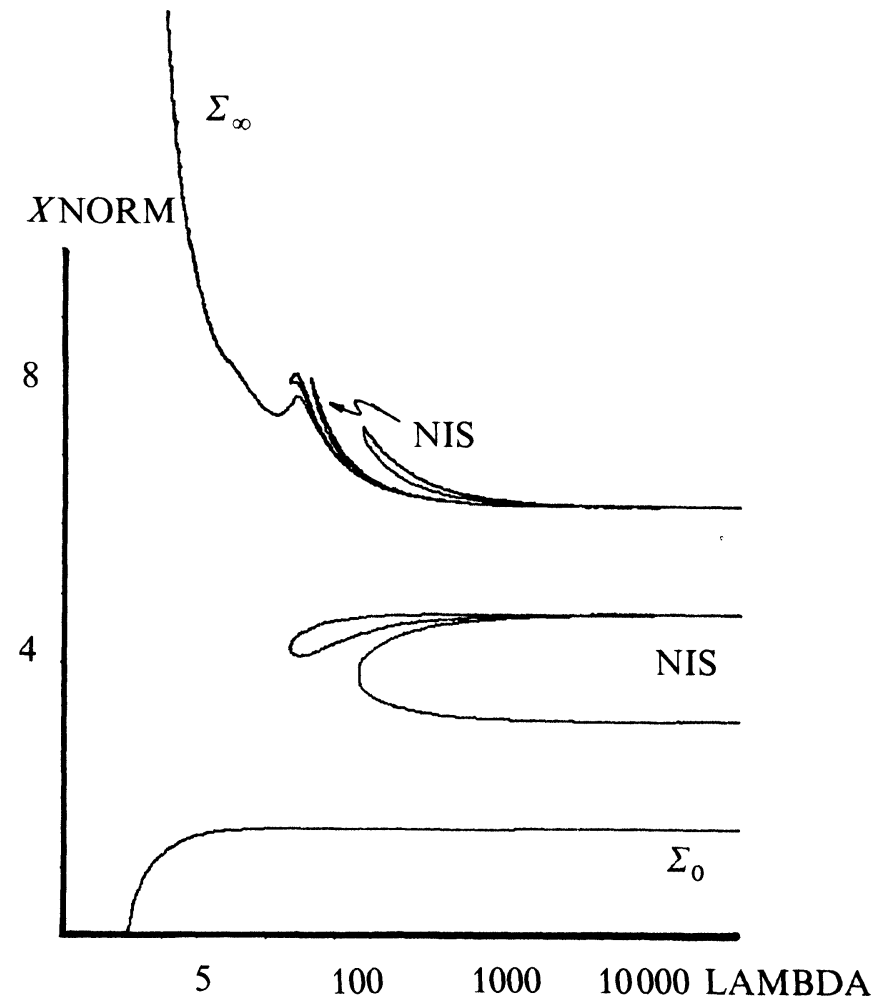

Figure 18

Again the picture suggests a correlation between the occurrence of NIS with that of a cusp in $\Sigma_{\infty}$.

Figure 19 is a closeup of Figure 18.

In Figures 20 to 26 we consider the same nonlinearity, however, only subject to a discretization on 2 internal meshpoints. This is done because a complete description of $\Phi^{-1}(0)$ can be given in this case.

According to our theoretical results of Sections 3 and 4 there will be at least 16 nontrivial solutions, these are shown in Figure 20.

The components of all solutions are $\left(x_{1}, x_{2}\right)$. Figure 21 gives a view from the $\left(x_{1}, x_{2}\right)$ plane towards $\lambda=\infty$. It shows the continua $\Sigma_{0}$ and $\Sigma_{\infty}$ together with 7 NIS continua. In Figure 22 we give a view from $\lambda=\infty$ onto the $\left(x_{1}, x_{2}\right)$ plane and recognize the 16 lattice points (zeros of the uncoupled system) to which all nontrivial solutions must converge as $\lambda \rightarrow \infty$.

In Theorem 3.3 we have studied the global perturbation $\Phi$ (see (5.5)) which is designed to provide a numerical access to $\Sigma_{\infty}$ from $\Sigma_{0}$. Figure 23 shows the $\Sigma_{0}$ component in $\Phi^{-1}(0)$ and hence shows that this has been achieved. The perturbation parameters $\mu_{3}$ and $\mu_{4}$ of Theorem 3.3 were chosen approximately 80,000. However, it should be observed, that $\Sigma_{0}$ and $\Sigma_{\infty}$ in Figure 23 are linked by one NIS component. Figure 24 shows $\Phi^{-1}(0)$ when Figure 23 is projected onto the $\left(\lambda, x_{2}\right)$ plane. 

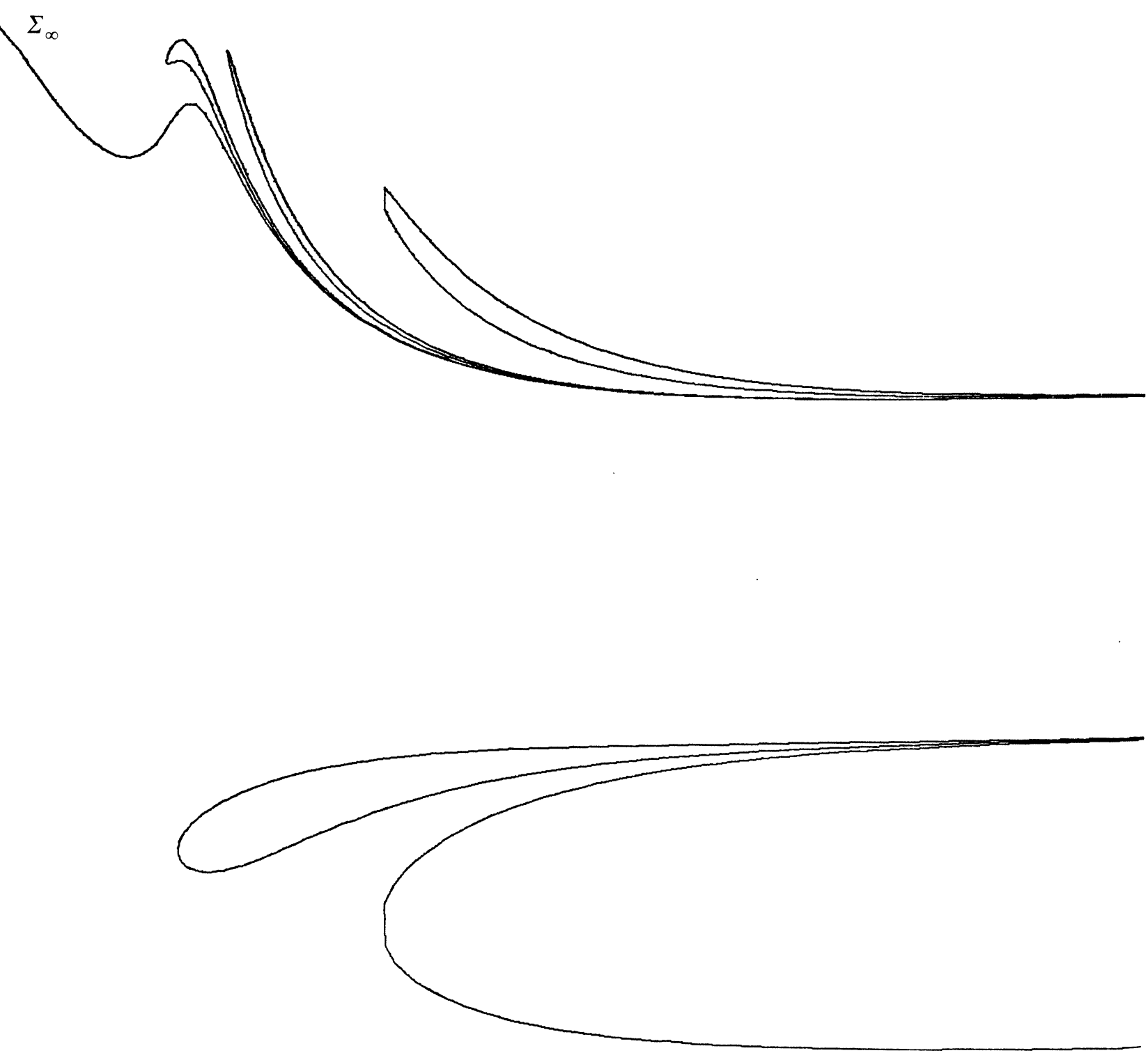

Figure 19

In Figures 25 and 26 we show the view of $\Phi^{-1}(0)$ of the $\Phi$-perturbation (5. 5) from $\lambda=80,000$ onto the $\left(x_{1}, x_{2}\right)$ plane. Figure 26 gives a complete picture of $\Phi^{-1}(0)$ whereas Figure 25 only shows the important portion of the continua in $\Phi^{-1}(0)$. In Figures 25 and 26 one observes that the $\Sigma_{0}$ component undergoes a bifurcation in the neighborhood of the point $(\pi, \pi)$. Hence in this specific example we have verified Theorem 3. 3 numerically but also observe that the $\Sigma_{0}$ component of $\Phi^{-1}(0)$ may be connected to several NIS components (here with three). 


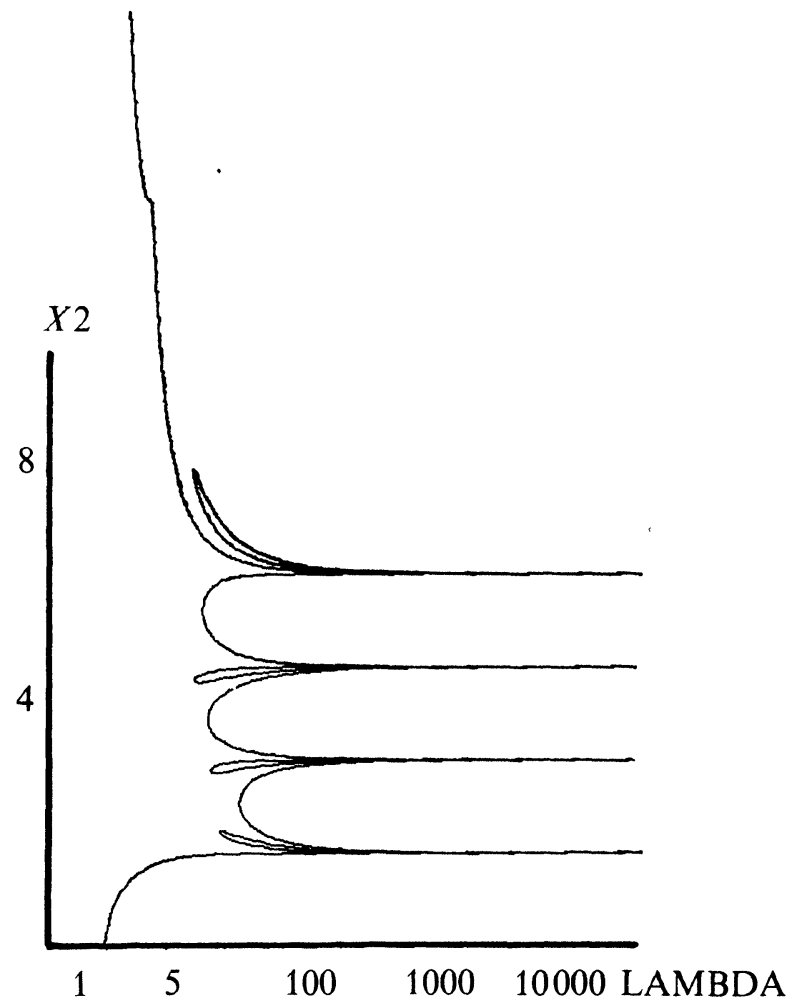

Figure 20

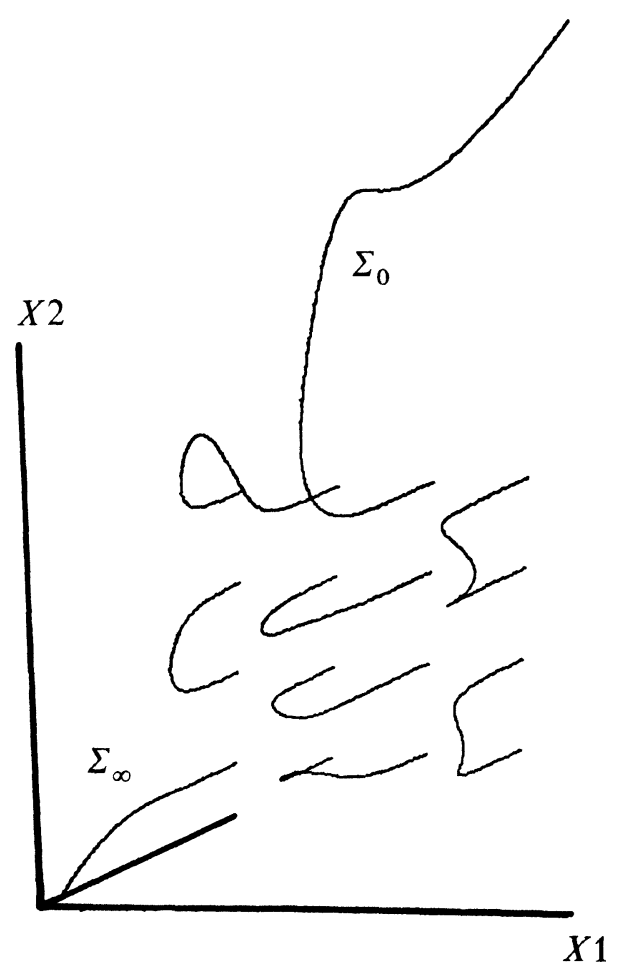

Figure 21 


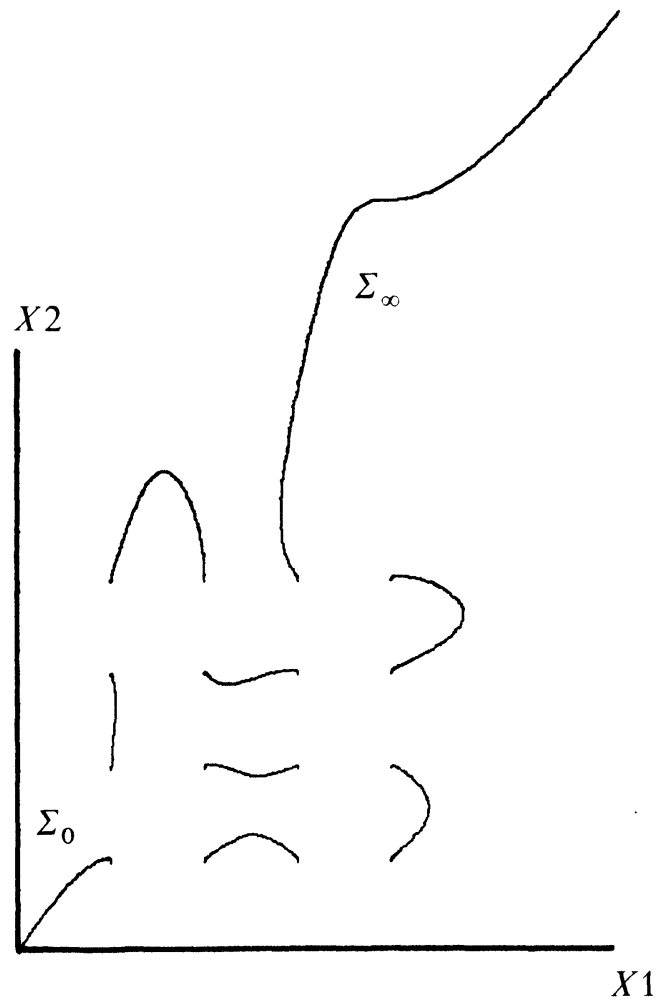

Figure 22

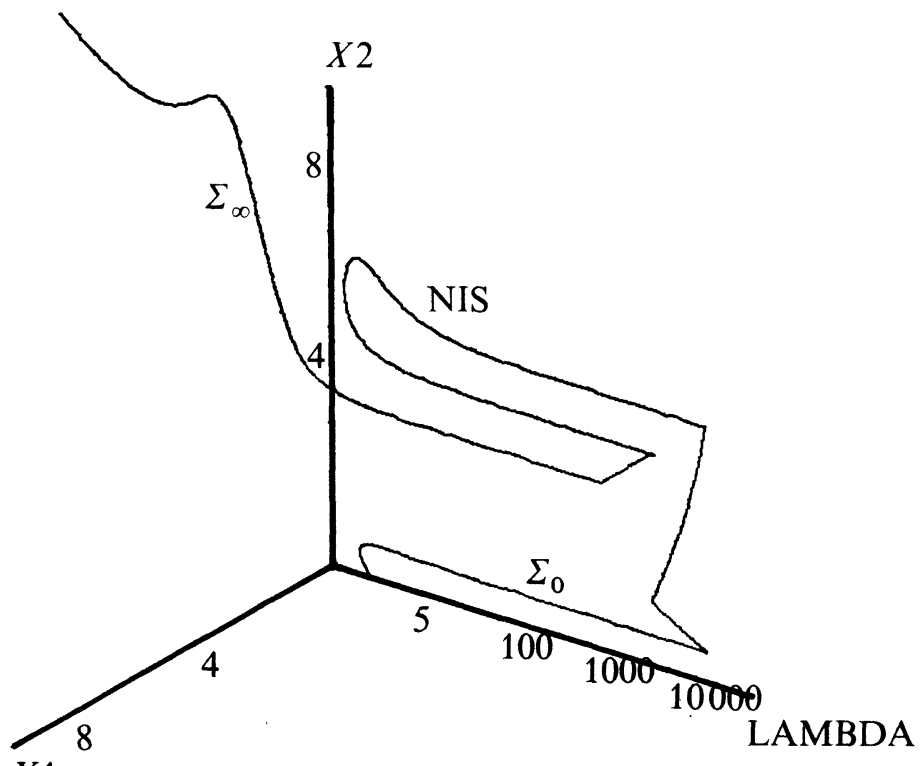

Figure 23 


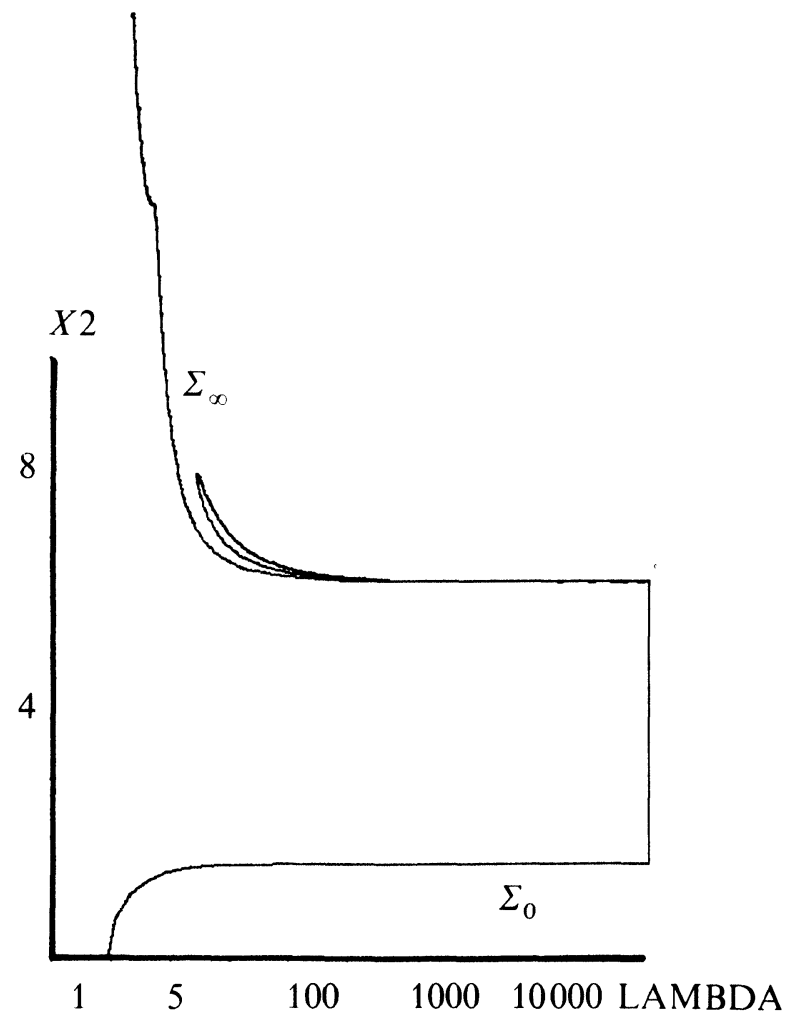

Figure 24

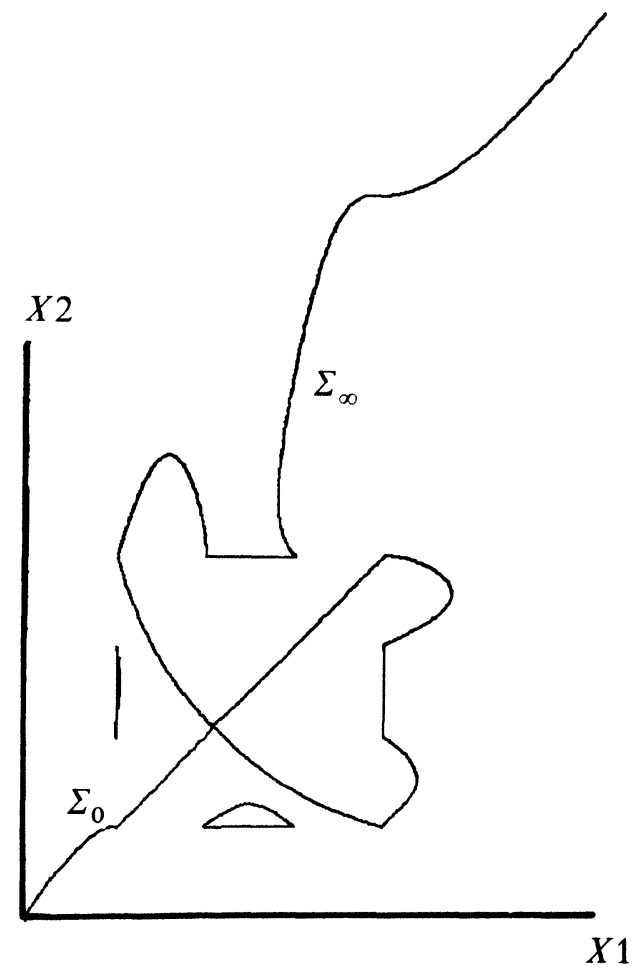

Figure 25 


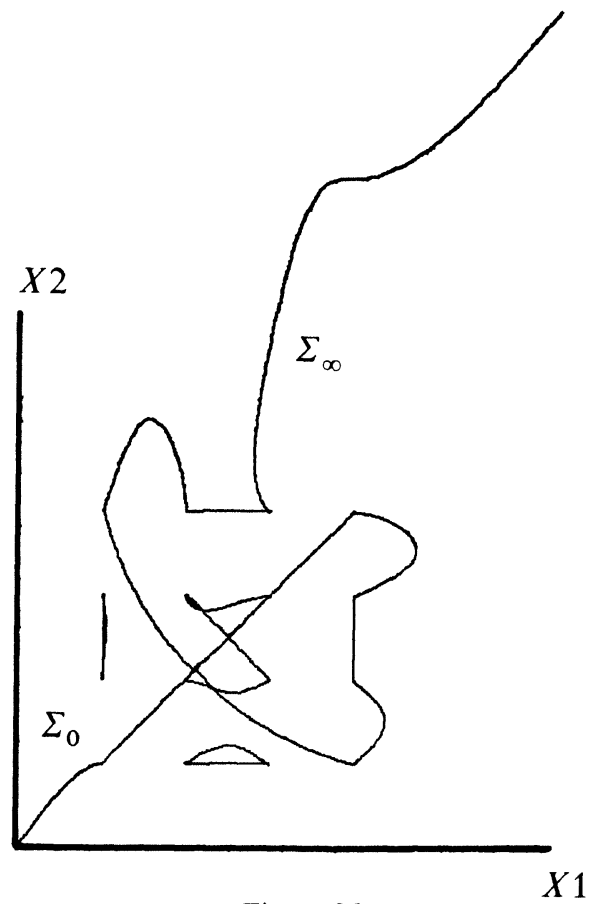

Figure 26

\section{$X 1$}

\section{The superlinear problem (4.5)}

For this particular boundary value problem we choose the nonlinearity $f(x)=x^{8}$ and base our finite difference approximation calculations on $n=3$ internal meshpoints.

It is well known that the boundary value problem (4.5) has a unique positive solution for all $\lambda>0$, and all $m>1$.

We study the finite difference approximation to (4. 5)

$$
A x=\lambda F(x)
$$

via an embedding into the two parameter problem

$$
\Phi(\lambda, \mu, x)=0,
$$

where

$$
\Phi(\lambda, \mu, x)=\left\{\begin{array}{l}
-\mu d+(1+\mu)[A x-\lambda F(x)],-1 \leqq \mu \leqq 0 \\
(1-\mu)[A x-\lambda F(x)]-\mu x, 0 \leqq \mu \leqq 1
\end{array}\right.
$$

and $d=\operatorname{col}(0,0,1)$.

According to Theorem 4.1, $\Phi^{-1}(0)$ undergoes a bifurcation from the zero solution for $\mu>0$ which provides an access to the positive NRS of (5: 7). There is also a continuum in $\Phi^{-1}(0)$ connecting the zero solution with another positive solution of (5.7) 
for $\mu<0$. The latter solution branch consists of NIS (non symmetric solutions) and is connected with a second NIS branch in $\Phi^{-1}(0)$ for $\mu>0$. This is a numerical verification of Theorem 4.6. Also it is verified that a homotopy of (5.7) with the problem

$$
d=0 \text { (no solution) }
$$

may lead to NIS. Figure 28 gives the solution surfaces of positive solutions of problem (5. 8).

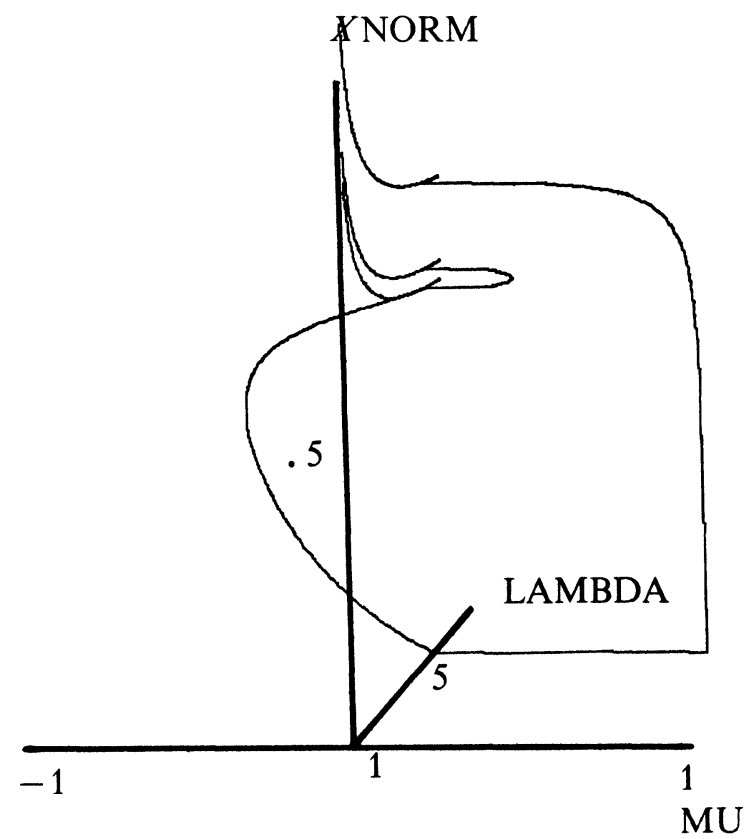

Figure 27

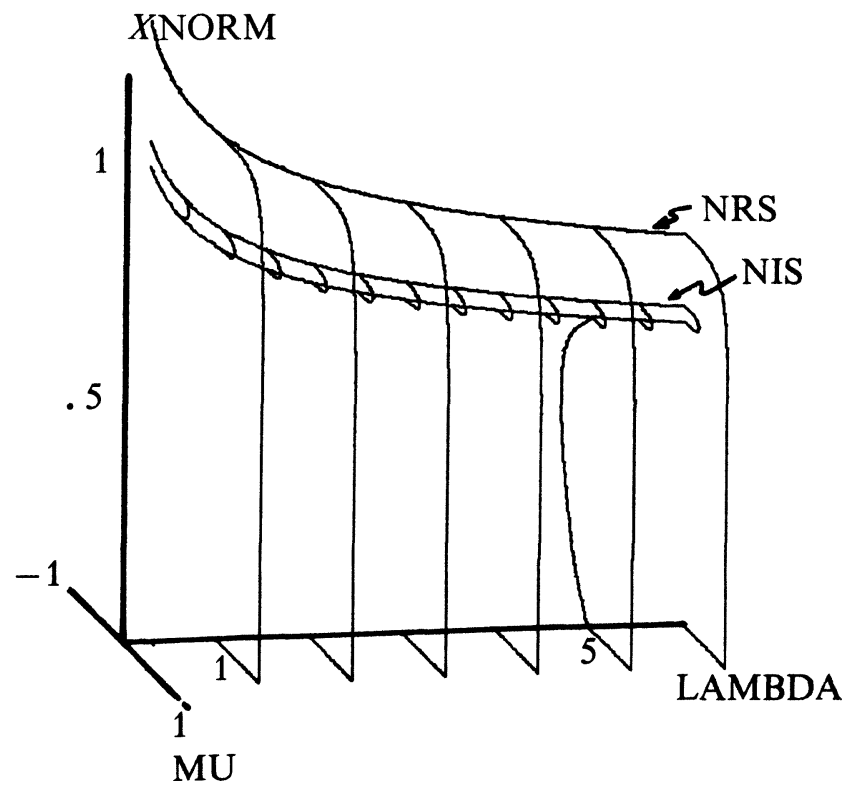

Figure 28 


\section{Numerical access to the Brown-Budin solutions}

In [5] it is shown that problem (1.3) may have positive solutions $u$ such that $z_{1} \leqq\|u\| \leqq z_{2 m}$ whenever $f \in \mathscr{F}$ and $G(u)=\int_{0}^{u} f(s) d s$ is such that there exists $i$ and $u^{*} \in\left[z_{2 i}, z_{2 i+1}\right]$ such that $G\left(u^{*}\right) \geqq G(u), 0 \leqq u \leqq u^{*}$. These solutions will lie on continua which, as has been pointed out in Section 3, must be disjoint from $\Sigma_{\infty}$ and $\Sigma_{0}$. In this final example we will show how such continua may be reached numerically using a global perturbation $\Phi$ like the one introduced in Theorem 3.3. Instead of considering this approach in great detail we merely indicate one procedure for tackling this problem.

Let $f_{m} \in \mathscr{F}$ be given. We choose two related nonlinearities $f$ and $g$ as in Figure 29 (the set of positive solutions of the corresponding boundary value problem is given below the graph of the nonlinearity):
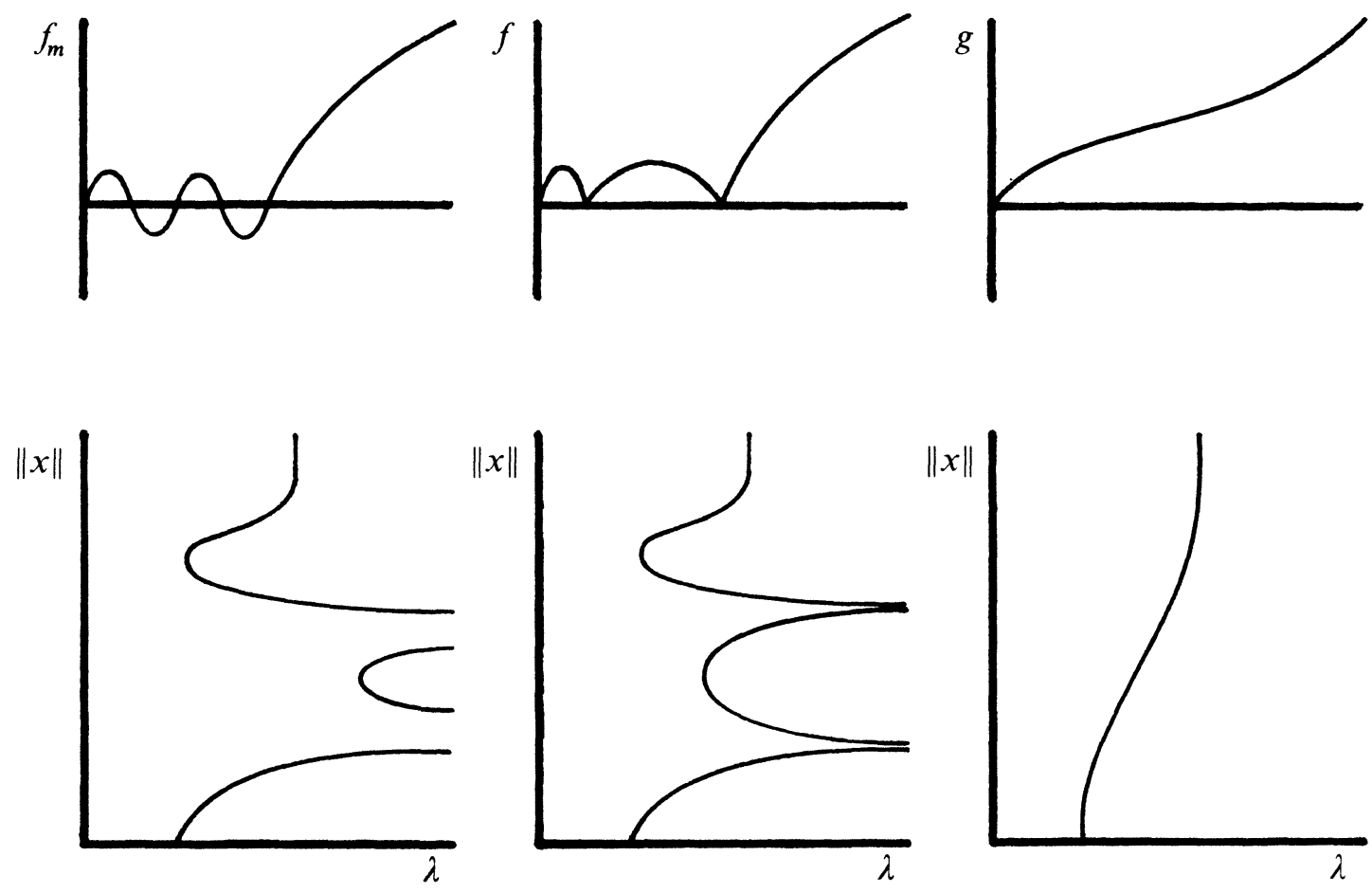

Figure 29

We next choose perturbation parameters $\mu_{1}<\mu_{2}<\mu_{3}<\mu_{4}$ as in the proof of Theorem 3. 3 and define the global perturbation $\Phi$ as we did there, i.e., connecting the problems

$$
A x-\lambda F_{m}(x)=0
$$

to $A x-\lambda F(x)=0$ and $A x-\lambda G(x)=0$ (where $F_{m}, F, G$ are again the Nemitskii operators of $f_{m}, f$, and $g$, respectively). Following the arguments in the proof of Theorem 3. 3 we then obtain a diagram like Figure 30 for $\Phi^{-1}(0)$. 


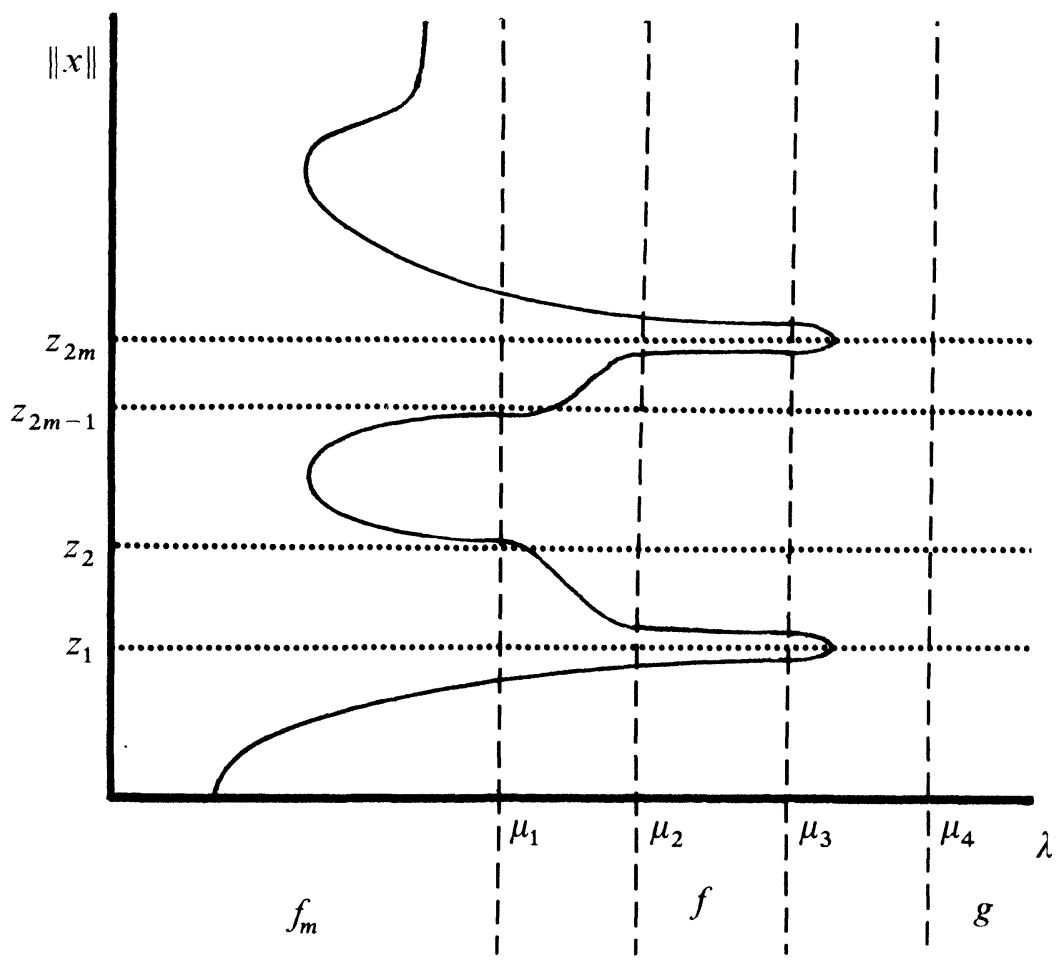

Figure 30

There are of course many other possible choices of the nonlinearities which may give other approaches to this problem.

\section{Concluding remarks and problems}

The discussion of the phase portraits (Figures 4-6) of problem (1.3) $\left(f=f_{m} \in \mathscr{F}\right)$ shows that in the finite difference approximation (3.2) those continua of type $\Sigma_{i}$ (see Theorem 3. 2) which are characterized by

$$
(x, \lambda) \in \Sigma_{i} \text { then } z_{2 j} \leqq x \leqq z_{2 j+1}, j<m
$$

may be NIS or NRS depending on $G(u)$,

$$
G(u)=\int_{0}^{u} f(s) d s .
$$

In [5] it is shown that under some assumptions on $G$ problem (1.3) has a continuum $\mathscr{C}$ of solutions satisfying conditions as in (6.1). This continuum will be approximated in each finite difference approximation (3.2), i.e. for each $n \in N$ ( $n=$ number of meshpoints) one will have a continuum $\Sigma_{i}(n)$ satisfying (6.1) which approximates $\mathscr{C}$ and, thus, the $\Sigma_{i}(n)$ will stabilize as $n \rightarrow \infty$. However, according to our theoretical analysis there will be additional continua of solutions of type $\Sigma_{i}$ in each finite difference approximation satisfying (6.1) and these will be NIS, i.e. as $n \rightarrow \infty$ these continua will vanish towards $\infty$. Thus, one observes two classes of continua of type $\Sigma_{i}$ which are not distinguished so far as solutions of (3.2) but can be classified with regard to the underlying differential equation. 
Problem 6. 2. (1) Is there an intrinsic characterization of NIS and NRS for problem (3. 2) with respect to the Brown-Budin solutions?

(2) What is the appropriate numerical procedure to compute the NRS in this context?

It is obvious from our analysis that nonlinear elliptic boundary value problems

$$
\left\{\begin{array}{cl}
L u=\lambda f(u) & \text { in } \Omega, \\
u=0 & \text { on } \partial \Omega
\end{array}\right.
$$

will have NIS for their finite difference approximations. However, phase plane arguments are not possible and therefore we have

Problem 6. 4. What is the appropriate method to distinguish NIS and NRS for finite difference approximations of (6. 3)?

In this paper we have restricted ourselves to Dirichlet problems. Obviously one should expect NIS also in presence of other boundary conditions (e.g. von Neumann or periodic boundary conditions). Again the problem will be to distinguish NIS from NRS with respect to the underlying differential equation.

More general problems of type

$$
\begin{cases}L u=\lambda f(u, \nabla u) & \text { in } \Omega, \\ B u=0 & \text { on } \partial \Omega\end{cases}
$$

are not immediately covered by our discussion. The reason is that the dependance on $\nabla u$ generates a coupling in $F(x)$, the discretization of $f$. A special problem related to this will be the investigation of Navier-Stokes problems with regard to NIS, where one is interested in particular for numerical solutions for $\lambda$ large $\left(\approx 10^{6}\right)$.

Another problem which ought to be mentioned here is that one should investigate whether the occurrance of NIS is typical for finite difference approximations only or may be also found for alternative numerical procedures (e.g. Galerkin methods).

\section{Added in proof.}

1) Problem (6.5) has been studied and partially answered in a recent paper by A. B. Stephens and G. R. Shubin [Multiple solutions and bifurcation of finite difference approximations to some steady problems of fluid dynamics, preprint Naval Surface Weapons Center]. These authors investigate the steady Burgers equation $u \cdot u_{x}-\lambda u_{x x}=0$ and obtain NIS solutions.

2) Recent numerical studies have shown that problem (5.1) admits at least three (structurally) different types of NIS. An analysis of these and a rigorous classification will appear in a forthcoming paper.

3) The perturbation first studied and used in section 3 has been exploited also to prove multiplicity results for nonlinear elliptic boundary value problems and nonlinear differential delay equations. For the first see H. O. Peitgen et K. Schmitt [Perturbations topologiques globales des problèmes non linéaires aux valeurs propres, C. R. Acad. Sc. Paris 291 (1980), 271-274]. For the latter see R. D. Nussbaum and H. O. Peitgen 
[Multiplicity results and the numerical approximation for special periodic solutions of $\dot{x}(t)=\lambda f(x(t-1))$, to appear]. Surprisingly, as this paper shows, one may have NIS also for numerical approximations of differential delay equations.

\section{Bibliography}

[1] E. Allgower, On a discretization of $y^{\prime \prime}+\lambda y^{k}=0$, Proc. Conf. Roy. Irish Acad., New York-London 1975.

[2] H. Amann, Fixed point equations and nonlinear eigenvalue problems in ordered Banach spaces, SIAM Review 18 (1976), 620-709.

[3] A. Ambrosetti and P. Hess, Positive solutions of asymptotically linear elliptic eigenvalue problems, J. Math. Anal. Appl. 73 (1980), 411-422.

[4] E. Bohl, On the bifurcation diagram of discrete analogues for ordinary bifurcation problems, Math. Meth. Appl. Sci. 1 (1979), 566-571.

[5] K. J. Brown and $H$. Budin, On the existence of positive solutions for a class of semilinear elliptic boundary value problems, SIAM J. Math. Anal. 10 (1979), 875-883.

[6] K. Deimling, Nichtlineare Gleichungen und Abbildungsgrade, Berlin-Heidelberg-New York 1974.

[7] J. Dugundji, Topology, Boston 1966.

[8] $R$. Gaines, Difference equations associated with boundary value problems for second order nonlinear ordinary differential equations, SIAM J. Num. Anal. 11 (1974), 411-434.

[9] H. Jürgens, H.-O. Peitgen and D. Saupe, Topological perturbations in the numerical study of nonlinear eigenvalue and bifurcation problems, Proceedings Symposium on Analysis and Computation of Fixed Points, S. M. Robinson (ed.), New York-London 1979.

[10] T. Y. Li and J. Yorke, Period three implies chaos, Amer. Math. Monthly 82 (1975), $985-992$.

[11] H.-O. Peitgen and M. Prüfer, The Leray-Schauder continuation method is a constructive element in the numerical study of nonlinear eigenvalue and bifurcation problems, Proc. Conf. Functional Differential Equations and Approximation of Fixed Points, Lecture Notes in Math. 730, Berlin-Heidelberg-New York 1980.

[12] P. H. Rabinowitz, Some aspects of nonlinear eigenvalue problems, Rocky Mountain J. Math. 3 (1973), $162-202$.

[13] K. Schmitt and H. L. Smith, On eigenvalue problems for nondifferentiable mappings, J. Diff. Equations 33 (1979), $294-319$.

[14] J. Schröder, M-matrices and generalizations using an operator theory approach, SIAM Review 20 (1978), $213-244$.

[15] R. S. Varga, Matrix Iterative Analysis, Englewood Cliffs, New Jersey 1962.

Forschungsschwerpunkt „Dynamische Systeme“, Fachbereich Mathematik, Universität Bremen, D-2800 Bremen Department of Mathematics, University of Utah, Salt Lake City, Utah 84112, USA 